\title{
The Diastase Content of Human Milk and its Relation to the Arakawa Reaction.
}

\author{
171st Report of the Peroxidase Reaction. \\ (118th Human Milk Study.) \\ By \\ Yadori Moriwaki. \\ (森 脇 苃) \\ (From the Department of Pediatrics, Faculty of Medicine, \\ Tohoku Imperial University, Sendai. \\ Director: Prof. A. Sato.)
}

It has been shown by numerous reports from our Laboratory that there is a wide fluctuation of different constituents of human milk according to the intensity of A rakawa's reaction, and it has been shown that, as a rule, "good" milk is positive* to A rakawa's reaction and that "bad" milk is negative to it (Cf. Reports of the Peroxidase Reaction). Now, it is well known that human milk contains several kinds of enzymes or substances presenting enzymic action. As to the relation between these enzymes and Arakawa's reaction, we see Takai's' ${ }^{1}$ work on salolase and Takamatsu's ${ }^{2)}$ and Orimo's) works on glyoxalase. Further, $\mathrm{Sato}^{4}$ studied the diastase content of urine of mothers in connexion with A rakawa's reaction. The latter three authors reported a remarkable increase of these enzymes in human milk or urine in

*. "Arakawa-positive milk" may be used in two different senses. One of these is : Arakawapositive in a biochemical sense. A sample of human milk is said to have become Arakawa-positive when it becomes blue on the addition of A rakawa's reagent. Here it means that the sample is not negative to A rakawa's reaction. The other of these two senses is : Arakawa-postive in a clinical sense. A sample of human milk is clinically Arakawa-positive only when it shows such a reaction as $\#$ or $\#$ in one minute of the addition of A rakawa's reagent. Another sample of human milk may be Arakawa-positive in the first described sense, but yet clinically negative. Normal Arakawa's reaction is of the intensity: (H) $1^{\prime}$ (\#) $2^{\prime}$ (\#) $3^{\prime}$ (\#) $4^{\prime}$ (\#) $5^{\prime}$ or of the stronger intensity.

1) S. Takai, Tohoku J. Exp. Med., 1936, 29, 82.

2) A. Takamatsu, Tohoku J. Exp. Med., 1938, 34, 509.

3) R. Orimo, Tohoku J. Exp. Med., 1939, 35, 34.

4) Sh. S a to, Tohoku J. Exp. Med., 1940, 37, 392. 
mothers with milk positive to Arakawa's reaction and a decrease of the enzymes in mothers with negative A rakawa's reaction. Salolase does not seem to have a very close relation to A rakawa's reaction.

In the present paper ${ }^{\dagger} \mathrm{I}$ want to report on the diastase content of human milk and its relation to Arakawa's reaction.

Before entering into main subject, I will here give a brief note of works on the milk diastase hitherto reported. Béchamp $\mathrm{p}^{5)}$ was the first author who found diastase in human milk, and his statement was accepted by many authors and numerous reports have been carried out on this subject. But within my knowledge, though I made a rather extensive survey over the literature, there are only a few reports as to the relation between milk diastase and milk vitamins outside as well as inside this country.

$\mathrm{Fujii}^{6)}$ saw an increase of milk diastase in the case of infantile beriberi in 1922 (though at that time the real etiology of beriberi still remained unknown). Chib could not reach any decisive conclusion as to the diastase content of the milk of cows fed on B-avitaminotic fodder. Of late, Sugie reported an increase of the diastase content of milk after vitamin $\mathrm{C}$ administration to mothers, though the number of cases were only 2-3. To my regret, I could not find any work concerning human milk diastase and vitamins except the above cited reports.

\section{Metbod and Materials.}

For the estimation of milk diastase, my own micro method devised for the purpose was used. The method (a New Micro Method for the Determination of Diastase in Human Milk; 25th Report on Micro Method) is described in a preceding paper'.

Specimens of human milk were mostly obtained from mothers who visited our Pediatric Dispensary, but in the case of those of colostrum, these were obtained from the Gynaecological and Obsterical Department* in this University. As to milk specimens of "healthy" mothers with "healthy" infants were gathered in a "Baby show" held on May 5th, 1934.

Test-tubes, disinfected and dried, were prepared for both breasts of the

+ Through this experiment of mine was performed at almost the same time (about 1933 1934) as Takai's work, the publication has been postponed for other reasons up to this day.

5) Béchamp, cit. W. Grimmer, Biochem. Ztschr., 1913, 53, 429.

6) S. Fuji i, Zika Zassi, 1922, No. 184, 754.

7) T. Chiba, Rinshoigaku, 1929, 17, 408.

8) Y.Sugie, Tokio Ijishinshi, 1938, No. 3130, 1057.

9) Y. Moriwaki, Tohoku J. Exp. Med.,

* Here I express my hearty thanks to Prof. Shinod a and doctors of the Gynaecological and Obsterial Department for the kindness shown toward me. 
same mother respectively. Breasts and nipples were cleaned and disinfected with alcohol to prevent possible contamination with infant's saliva which might have more or less diastatic action. A rakawa's reaction, immediately after milk samples were obtained, was examined using part of each sample.

\section{Results of Experiment.}

1. Individual difference and mean value of diastase content in human milk (Cf. Tables I and II).

Of 380 different breasts, milk diastase was 1.8 in the minimum and 150.0 in the maximum. The mean was 21.6.

TABle I.

All the cases examined. (1)

\begin{tabular}{|c|c|c|c|c|c|c|}
\hline No. & Name & $\begin{array}{l}\text { Ara- } \\
\text { kawa's } \\
\text { reaction }\end{array}$ & $\begin{array}{c}\text { Diastase } \\
\text { content }\end{array}$ & $\begin{array}{c}\text { Age of } \\
\text { children } \\
\mathrm{m}=\text { months } \\
\text { age of } \\
\text { mothers } \\
\mathrm{y}=\text { years }\end{array}$ & $\begin{array}{c}\text { Condition } \\
\text { of } \\
\text { secretion }\end{array}$ & Diagnosis \\
\hline $\begin{array}{l}1 \\
2 \\
\end{array}$ & K. U: & $\begin{array}{l}10(-) \\
12(-)\end{array}$ & $\begin{array}{l}25.0 \\
30.0\end{array}$ & $\begin{array}{l}14 \mathrm{~m} \\
34 \mathrm{y}\end{array}$ & $\mathrm{R}=\mathrm{L}$ & Atrophy \\
\hline $\begin{array}{l}3 \\
4 \\
\end{array}$ & K. H. & $\begin{array}{l}7(+) \\
1(+)\end{array}$ & $\begin{array}{l}60.2 \\
14.2 \\
\end{array}$ & $\begin{array}{l}15 \mathrm{~m} \\
28 \mathrm{y}\end{array}$ & $\mathrm{R}<\mathrm{L}$ & Dyspepsia \\
\hline $\begin{array}{l}5 \\
6 \\
\end{array}$ & R. H. & $\begin{array}{l}5(H) \\
1(H)\end{array}$ & $\begin{array}{l}14.8 \\
10.0\end{array}$ & $\underset{*}{11 \mathrm{~m}}$ & $\mathbf{R}=\mathbf{L}$ & Whooping cough \\
\hline $\begin{array}{l}7 \\
8\end{array}$ & Y. K. & $\begin{array}{l}1(H) \\
2(H)\end{array}$ & $\begin{array}{l}13.6 \\
13.6\end{array}$ & $12 \mathrm{~m}$ & $\mathrm{R}=\mathrm{L}$ & $*$ \\
\hline $\begin{array}{r}9 \\
10\end{array}$ & E. F. & $\begin{array}{l}1(+4) \\
7(+)\end{array}$ & $\begin{array}{l}13.6 \\
18.8 \\
\end{array}$ & $\begin{array}{l}4 \mathrm{~m} \\
24 \mathrm{y}\end{array}$ & $\mathrm{R}=\mathrm{L}$ & Dyspepsia \\
\hline $\begin{array}{l}11 \\
12\end{array}$ & Y. O. & $\begin{array}{l}11( \pm) \\
10(-)\end{array}$ & $\begin{array}{l}18.8 \\
13.6 \\
\end{array}$ & $\begin{array}{l}19 \mathrm{~m} \cdot \\
32 \mathrm{y}\end{array}$ & $\mathrm{R}=\mathrm{L}$ & Angina \\
\hline $\begin{array}{l}13 \\
14\end{array}$ & K. N. & $\begin{array}{l}1(H) \\
3(-)\end{array}$ & $\begin{array}{l}11.5 \\
42.9\end{array}$ & $\begin{array}{l}11 \mathrm{~m} \\
25 \mathrm{y}\end{array}$ & $\mathrm{R}>\mathrm{L}$ & Acute dyspepsia \\
\hline $\begin{array}{l}15 \\
16 \\
\end{array}$ & N. M. & $\begin{array}{l}16(-) \\
16(-)\end{array}$ & $\begin{array}{l}60.0 \\
60.0 \\
\end{array}$ & $\begin{array}{r}3 \mathrm{~m} \\
25 \mathrm{y} \\
\end{array}$ & $\mathrm{R}=\mathbf{L}$ & Acute dyspepsia $f$ atrophy \\
\hline $\begin{array}{l}17 \\
18\end{array}$ & T. B. & $\left|\begin{array}{c}+H+H \\
1(+)\end{array}\right|$ & $\begin{array}{l}9.4 \\
7.5\end{array}$ & $\begin{array}{r}1 \mathrm{~m} \\
31 \mathrm{y}\end{array}$ & $\mathrm{R}=\mathrm{L}$ & Healthy \\
\hline $\begin{array}{l}19 \\
20\end{array}$ & M. K. & $\begin{array}{l}1(H) \\
4(+)\end{array}$ & $\begin{array}{l}16.6 \\
12.2\end{array}$ & $\begin{array}{r}9 \mathrm{~m} \\
34 \mathrm{y}\end{array}$ & $\mathbf{R}=\mathbf{L}$ & Paresis of neck muscles \\
\hline $\begin{array}{l}21 \\
22\end{array}$ & C. $\mathrm{K}$. & $\begin{array}{l}1(H) \\
1(H)\end{array}$ & $\begin{array}{r}60.0 \\
2.5\end{array}$ & $\begin{array}{c}4 \mathrm{~m} \\
*\end{array}$ & $\mathrm{R}>\mathrm{L}$ & Torticollis \\
\hline $\begin{array}{l}23 \\
24\end{array}$ & S. O. & $\begin{array}{r}1(\mathrm{H}) \\
+\mathrm{HH} H \mathrm{H}\end{array}$ & $\begin{array}{l}10.0 \\
10.7\end{array}$ & $\begin{array}{l}11 \mathrm{~m} \\
26 \mathrm{y}\end{array}$ & $\mathrm{R}=\mathrm{L}$ & Atrophy with dyspepsia \\
\hline $\begin{array}{l}25 \\
26\end{array}$ & T. K. & $\begin{array}{l}1(H) \\
1(H)\end{array}$ & $\begin{array}{l}11.5 \\
16.6\end{array}$ & $\begin{array}{r}9 \mathrm{~m} \\
24 \mathrm{y}\end{array}$ & $\mathrm{R}=\mathrm{L}$ & Dyspepsia \\
\hline $\begin{array}{l}27 \\
28\end{array}$ & K. S. & $\begin{array}{c}-+++H \\
12(-)\end{array}$ & $\begin{array}{l}1.8 \\
1.8\end{array}$ & $\begin{array}{r}4 \mathrm{~m} \\
21 \mathrm{y}\end{array}$ & $\mathrm{R}=\mathrm{L}$ & Hypotrophy \\
\hline $\begin{array}{l}29 \\
30\end{array}$ & K. K. & $\begin{array}{c}15( \pm) \\
4(+)\end{array}$ & $\begin{array}{l}25.0 \\
17.6\end{array}$ & $\begin{array}{r}2 \mathrm{~m} \\
22 \mathrm{y}\end{array}$ & $\mathrm{R}<\mathrm{L}$ & Healthy \\
\hline
\end{tabular}

$\dagger$ Colostrum and milk obtained in a "Baby show" were omitted. Moreover, in some cases milk of the same lactating mothers was examined repeatedly in different occasions. In such a case, of course, only one instance out of several was used in my statistics in the present paper. 
Table

\begin{tabular}{|c|c|c|c|c|c|c|}
\hline No. & Name & $\begin{array}{l}\text { Ara- } \\
\text { kawa's } \\
\text { reaction }\end{array}$ & $\begin{array}{l}\text { Diastase } \\
\text { content }\end{array}$ & $\begin{array}{c}\text { Age of } \\
\text { children } \\
\text { m=months } \\
\text { age of } \\
\text { mothers } \\
\mathrm{y}=\text { years }\end{array}$ & $\begin{array}{l}\text { Condition } \\
\text { of } \\
\text { secretion }\end{array}$ & Diagnosis \\
\hline $\begin{array}{l}31 \\
32 \\
\end{array}$ & T. A. & $\begin{array}{c}1(+4) \\
+H H+H \\
\end{array}$ & $\begin{array}{l}7.5 \\
3.5 \\
\end{array}$ & $\begin{array}{l}17 \mathrm{~m} \\
34 \mathrm{y}\end{array}$ & $\mathrm{R}=\mathrm{L}$ & Dyspepsia $†$ rhinopharyngitis \\
\hline $\begin{array}{l}33 \\
34 \\
\end{array}$ & I. M. & $\begin{array}{l}1(\#) \\
1(\#) \\
\end{array}$ & $\begin{array}{l}9.4 \\
2.5 \\
\end{array}$ & $\begin{array}{r}7 \mathrm{~m} \\
25 \mathrm{y}\end{array}$ & $\mathrm{R}=\mathrm{L}$ & Chronic dyspepsia \\
\hline $\begin{array}{l}35 \\
36 \\
\end{array}$ & M. I. & $\left|\begin{array}{c}16(-) \\
--+++\mid\end{array}\right|$ & $\begin{array}{l}15.8 \\
33.4\end{array}$ & $\begin{array}{l}5 \mathrm{~m} \\
35 \mathrm{y}\end{array}$ & $\mathrm{R}=\mathrm{L}$ & $\begin{array}{l}\text { Severe dyspepsia } f \text { decompo- } \\
\text { sition }\end{array}$ \\
\hline $\begin{array}{l}37 \\
38 \\
\end{array}$ & S. W. & $\begin{array}{l}1(\mathrm{H}) \\
1(\mathrm{H})\end{array}$ & $\begin{array}{l}2.5 \\
2.5 \\
\end{array}$ & $\begin{array}{r}6 \mathrm{~m} \\
38 \mathrm{y} \\
\end{array}$ & $\mathrm{R}=\mathrm{L}$ & $\begin{array}{l}\text { Chronic dyspepsia } f \text { pharyn- } \\
\text { gitis }\end{array}$ \\
\hline $\begin{array}{l}39 \\
40\end{array}$ & N. T. & $\begin{array}{r}4(-) \\
10(-) \\
\end{array}$ & $\begin{array}{l}42.8 \\
37.5 \\
\end{array}$ & $\begin{array}{r}1 \mathrm{~m} \\
33 \mathrm{y}\end{array}$ & $\mathrm{R}>\mathrm{L}$ & Osteochondritis syphilitica \\
\hline $\begin{array}{l}41 \\
42\end{array}$ & S. I. & $\begin{array}{l}7( \pm) \\
2(+)\end{array}$ & $\begin{array}{l}8.8 \\
8.3\end{array}$ & $\begin{array}{l}14 \mathrm{~m} \\
31 \mathrm{y}\end{array}$ & $\mathrm{R}=\mathrm{L}$ & Hypotrophy \\
\hline $\begin{array}{l}43 \\
44 \\
\end{array}$ & T. S. & $\begin{array}{l}1(H) \\
7(+) \\
\end{array}$ & $\begin{array}{l}21.5 \\
18.8 \\
\end{array}$ & $\begin{array}{l}11 \mathrm{~m} \\
25 \mathrm{y}\end{array}$ & $\mathrm{R}>\mathrm{I}$ & Eczema $\uparrow$ stomatitis \\
\hline $\begin{array}{l}45 \\
46 \\
\end{array}$ & A. S. & $\begin{array}{c}16(-) \\
\pm H+H+H\end{array}$ & $\begin{array}{l}66.7 \\
28.1 \\
\end{array}$ & $\begin{array}{r}4 \mathrm{~m} \\
27 \mathrm{y} \\
\end{array}$ & $\mathrm{R}=\mathrm{L}$ & Obstipation by B-avitaminosis \\
\hline $\begin{array}{l}47 \\
48 \\
\end{array}$ & S. K. & \begin{tabular}{l|}
1 (\#) \\
$1($ \#) \\
\end{tabular} & $\begin{array}{l}10.7 \\
11.5 \\
\end{array}$ & $\begin{array}{r}4 \mathrm{~m} \\
37 \mathrm{y}\end{array}$ & $\mathrm{R}=\mathrm{L}$ & Dyspepsia \\
\hline $\begin{array}{l}49 \\
50 \\
\end{array}$ & T. M. & $\begin{array}{l}1(\text { H) } \\
1(\#)\end{array}$ & $\begin{array}{l}9.4 \\
9.4 \\
\end{array}$ & $\begin{array}{r}7 \mathrm{~m} \\
23 \mathrm{y} \\
\end{array}$ & $R=L_{p}$ & Dyspepsia \\
\hline $\begin{array}{l}51 \\
52 \\
\end{array}$ & T. F. & $\begin{array}{l}1(\#) \\
1(\#)\end{array}$ & $\begin{array}{l}26.5 \\
20.0 \\
\end{array}$ & $\begin{array}{l}18 \mathrm{~m} \\
28 \mathrm{y}\end{array}$ & $\mathrm{R}=\mathrm{L}$ & Influenza \\
\hline $\begin{array}{l}53 \\
54 \\
\end{array}$ & K. M. & $\begin{array}{l}1(\text { (H) } \\
1(\text { \#) }\end{array}$ & $\begin{array}{l}12.2 \\
13.6 \\
\end{array}$ & $\begin{array}{l}13 \mathrm{~m} \\
26 \mathrm{y}\end{array}$ & - $\mathrm{R}=\mathrm{L}_{\mathrm{p}}$ & Dystrophy \\
\hline $\begin{array}{l}55 \\
56 \\
\end{array}$ & T. M. & $\begin{array}{l}16(-) \\
16(-)\end{array}$ & $\begin{array}{r}6.0 \\
12.2 \\
\end{array}$ & $\begin{array}{r}8 \mathrm{~m} \\
21 \mathrm{y} \\
\end{array}$ & $\mathrm{R}=\mathrm{L}$ & Dystrophy \\
\hline $\begin{array}{l}57 \\
58 \\
\end{array}$ & Y.I. & $\begin{array}{l}1(\#) \\
1(\#) \\
\end{array}$ & $\begin{array}{l}20.0 \\
12.0 \\
\end{array}$ & $\begin{array}{r}7 \mathrm{~m} \\
29 . \mathrm{y} \\
\end{array}$ & $R=L$ & Dyspepsia \\
\hline $\begin{array}{l}59 \\
60 \\
\end{array}$ & Y. T. & $\begin{array}{c}-++t+1 \\
7(+)\end{array}$ & $\begin{array}{r}2.5 \\
2.5 \\
\end{array}$ & $\begin{array}{l}15 \mathrm{~m} \\
27 \mathrm{y}\end{array}$ & $\mathrm{R}=\mathrm{L}_{\mathrm{p}}$ & Stomatitis \\
\hline $\begin{array}{l}61 \\
62 \\
\end{array}$ & K. K. & $\begin{array}{r}6(-) \\
14(-)\end{array}$ & $\begin{array}{l}33.4 \\
33.4 \\
\end{array}$ & $\begin{array}{r}8 \mathrm{~m} \\
21 \mathrm{y}\end{array}$ & $\mathrm{R}=\mathrm{L}$ & Retention of bladder \\
\hline $\begin{array}{l}63 \\
64 \\
\end{array}$ & X. Y. & $\begin{array}{l}5( \pm) \\
3( \pm)\end{array}$ & $\begin{array}{l}17.6 \\
17.6 \\
114\end{array}$ & $\begin{array}{l}5 \mathrm{~m} \\
* \\
\end{array}$ & * & * \\
\hline $\begin{array}{l}65 \\
66 \\
\end{array}$ & Y. M. & $\begin{array}{c}5( \pm) \\
\pm++H+H\end{array}$ & $\begin{array}{l}11.1 \\
10.0 \\
\end{array}$ & $5 \mathrm{~m}$ & $\mathrm{R}=\mathrm{L}$ & Infantile preberibeti \\
\hline $\begin{array}{l}67 \\
68 \\
\end{array}$ & M. S. & $\begin{array}{l}1(\mathrm{H}) \\
1(\mathrm{H}) \\
\end{array}$ & $\begin{array}{l}13.6 \\
15.8 \\
200\end{array}$ & $\begin{array}{r}9 \mathrm{~m} \\
29 \mathrm{y} \\
\end{array}$ & $R=L$ & Poliomyelitis anterior acuta \\
\hline $\begin{array}{l}69 \\
70 \\
\end{array}$ & Y.U. & $\begin{array}{l}1(+) \\
1(+\#)\end{array}$ & $\begin{array}{l}20.0 \\
17.6 \\
\end{array}$ & $\begin{array}{l}* \\
* \\
\end{array}$ & $*$ & Healthy \\
\hline $\begin{array}{l}71 \\
72 \\
\end{array}$ & J. S. & $\begin{array}{l}1(\#) \\
5(H) \\
\end{array}$ & $\begin{array}{l}15.0 \\
20.0 \\
\end{array}$ & $\begin{array}{r}9 \mathrm{~m} \\
24 \mathrm{y} \\
\end{array}$ & * & Acute dyspepsia $†$ angina \\
\hline $\begin{array}{l}73 \\
74 \\
\end{array}$ & K. S. & $\begin{array}{l}1(+4) \\
5(+)\end{array}$ & $\begin{array}{l}23.1 \\
20.0 \\
\end{array}$ & $\begin{array}{c}17 \mathrm{~m} \\
* \\
\end{array}$ & $\mathrm{R}=\mathrm{L}$ & Acute dyspepsia \\
\hline $\begin{array}{l}75 \\
76 \\
\end{array}$ & K. K. & $\begin{array}{l}1(+) \\
4(+)\end{array}$ & $\begin{array}{l}10.0 \\
10.0 \\
\end{array}$ & $\begin{array}{c}34 \mathrm{~m} \\
* \\
\end{array}$ & $\mathrm{R}=\mathrm{L}$ & Nocturnal enuresis \\
\hline $\begin{array}{l}77 \\
78 \\
\end{array}$ & M. T. & $\begin{array}{l}1(+) \\
1(+) \\
\end{array}$ & $\begin{array}{l}28.1 \\
32.1 \\
\end{array}$ & $\begin{array}{l}12 \mathrm{~m} \\
29 \mathrm{y}\end{array}$ & $\mathrm{R}=\mathrm{L}_{\mathrm{p}}$ & Dystrophy $\uparrow$ pharyngitis \\
\hline $\begin{array}{l}79 \\
80 \\
81\end{array}$ & S. A. & $\begin{array}{l}11(-) \\
12(-) \\
4(+)\end{array}$ & $\begin{array}{l}32.1 \\
28.5 \\
\end{array}$ & $\begin{array}{r}3 \mathrm{~m} \\
22 \mathrm{y} \\
\end{array}$ & $\mathrm{R}=\mathrm{L}$ & Infantile beriberi \\
\hline $\begin{array}{l}81 \\
82\end{array}$ & E. $O$. & $\begin{array}{l}4( \pm) \\
1(+)\end{array}$ & $\begin{array}{l}21.5 \\
15.0\end{array}$ & $\begin{array}{l}12 \mathrm{~m} \\
30 \mathrm{y}\end{array}$ & $R>L$ & Dyspepsia $†$ pharyngitis \\
\hline
\end{tabular}


I. Continued (2).

\begin{tabular}{|c|c|c|c|c|c|c|}
\hline No & Name & $\begin{array}{l}\text { Ara- } \\
\text { kawa's } \\
\text { reaction }\end{array}$ & $\begin{array}{l}\text { Diastase } \\
\text { content }\end{array}$ & $\begin{array}{c}\text { Age of } \\
\text { children } \\
\text { m=montbs } \\
\text { age of } \\
\text { mothers } \\
\mathrm{y}=\text { years }\end{array}$ & $\begin{array}{l}\text { Condition } \\
\text { of } \\
\text { secretion }\end{array}$ & Diagnosis \\
\hline $\begin{array}{l}83 \\
84\end{array}$ & H. I. & $\begin{array}{l}4( \pm) \\
5(H)\end{array}$ & $\begin{array}{r}11.1 \\
7.5\end{array}$ & $5 \mathrm{~m}$ & $\mathrm{R}=\mathrm{L}$ & Rhinopharyngitis \\
\hline $\begin{array}{l}85 \\
86\end{array}$ & Z. T. & $\begin{array}{l}6(+) \\
5(H)\end{array}$ & $\begin{array}{l}27.6 \\
15.0\end{array}$ & $\begin{array}{l}14 \mathrm{~m} \\
26 \mathrm{y}\end{array}$ & $\mathrm{R}=\mathrm{L}$ & Poliomyelitis anterior acuta \\
\hline $\begin{array}{l}87 \\
88\end{array}$ & T. $\mathrm{K}$. & $\begin{array}{l}1(H) \\
1(H)\end{array}$ & $\begin{array}{l}11.5 \\
12.0\end{array}$ & $\begin{array}{l}11 \mathrm{~m} \\
37 \mathrm{y}\end{array}$ & $\mathrm{R}=\mathrm{L}$ & Pyelocytitis \\
\hline $\begin{array}{l}89 \\
90\end{array}$ & H. U. & $\begin{array}{r}4(-) \\
14(-)\end{array}$ & $\begin{array}{l}23.1 \\
21.5\end{array}$ & $\begin{array}{r}2 \mathrm{~m} \\
27 \mathrm{y}\end{array}$ & $\mathrm{R}=\mathrm{L}$ & Infantile preberiberi \\
\hline $\begin{array}{l}91 \\
92\end{array}$ & K. S. & $\begin{array}{l}1(+) \\
1(+)\end{array}$ & $\begin{array}{l}16.6 \\
18.8\end{array}$ & $\begin{array}{l}13 \mathrm{~m} \\
41 \mathrm{y}\end{array}$ & $\mathrm{R}=\mathrm{L}_{\mathrm{p}}$ & Dyspepsia + hereditary syphilis \\
\hline $\begin{array}{r}93 \\
94\end{array}$ & K. S. & $\mid \begin{array}{c} \pm+H+\# \\
5( \pm)\end{array}$ & $\begin{array}{l}95.0 \\
95.0\end{array}$ & $1_{*}^{1 \mathrm{~m}}$ & $\mathrm{R}=\mathrm{L}$ & Periproctitis + dyspepsia \\
\hline $\begin{array}{l}95 \\
96\end{array}$ & K. I. & $\begin{array}{r}11(+) \\
7( \pm)\end{array}$ & $\begin{array}{l}20.0 \\
17.6\end{array}$ & $\begin{array}{l}22 \mathrm{~m} \\
25 \mathrm{y}\end{array}$ & $\mathrm{R}>\mathrm{L}_{\mathrm{p}}$ & Dyspepsia \\
\hline $\begin{array}{l}97 \\
98 \\
\end{array}$ & E. $O$. & $\begin{array}{l}3( \pm) \\
1(+H)\end{array}$ & $\begin{array}{l}40.0 \\
40.0\end{array}$ & $\begin{array}{l}12 \mathrm{~m} \\
24 \mathrm{y}\end{array}$ & $\mathrm{R}=\mathrm{L}$ & Dyspepsia \\
\hline $\begin{array}{r}99 \\
100 \\
\end{array}$ & S. 0 . & $\begin{array}{l}5(H) \\
5(+4)\end{array}$ & $\begin{array}{l}24.0 \\
26.1\end{array}$ & $\begin{array}{l}13 \mathrm{~m} \\
29 \mathrm{y}\end{array}$ & $\mathrm{R}=\mathbf{L}$ & Dyspepsia \\
\hline $\begin{array}{l}101 \\
102 \\
\end{array}$ & K. S. & $\begin{array}{l}2(+) \\
2(+)\end{array}$ & $\begin{array}{l}5.0 \\
5.0 \\
\end{array}$ & $\begin{array}{r}3 \mathrm{~m} \\
29 . \mathrm{y} \\
\end{array}$ & $\mathbf{R}=\mathbf{L}$ & Dyspepsia \\
\hline $\begin{array}{l}103 \\
104\end{array}$ & T. K. & $\begin{array}{l}2(+) \\
4(+)\end{array}$ & $\begin{array}{l}27.6 \\
27.6\end{array}$ & $\begin{array}{l}4 \mathrm{~m} \\
25 \mathrm{y}\end{array}$ & $\mathrm{R}=\mathrm{L}$ & Angina + Infantile beriberi \\
\hline $\begin{array}{l}105 \\
106\end{array}$ & F. K. & $\begin{array}{l}4(H) \\
1(H)\end{array}$ & $\begin{array}{l}11.5 \\
12.5\end{array}$ & $\begin{array}{r}3 \mathrm{~m} \\
34 \mathrm{y}\end{array}$ & $\mathbf{R}=\mathbf{L}$ & Acute rhinitis \\
\hline $\begin{array}{l}107 \\
108\end{array}$ & S. T. & $\begin{array}{l}1(+) \\
2(+)\end{array}$ & $\begin{array}{l}35.5 \\
56.4 \\
\end{array}$ & $\begin{array}{c}2 \mathrm{~m} \\
26 \mathrm{y}\end{array}$ & $\mathrm{R}^{>} \mathrm{L}_{\mathrm{p}}$ & Dyspepsia \\
\hline $\begin{array}{l}109 \\
110\end{array}$ & K. A. & $\begin{array}{l}1(+) \\
2(+)\end{array}$ & $\begin{array}{l}37.5 \\
46.0\end{array}$ & $\begin{array}{l}3 \mathrm{~m} \\
29 \mathrm{y}\end{array}$ & $\mathrm{R}>\mathrm{L}_{\mathrm{p}}$ & Infantile preberiberi \\
\hline $\begin{array}{l}111 \\
112\end{array}$ & M. T. & $\begin{array}{l}15(-) \\
16(-)\end{array}$ & $\begin{array}{l}25.0 \\
27.6\end{array}$ & $\begin{array}{r}1 \mathrm{~m} \\
29 \mathrm{y}\end{array}$ & $\mathrm{R}=\mathrm{L}$ & Infantile preberiberi \\
\hline $\begin{array}{l}113 \\
114 \\
\end{array}$ & T. $O$. & $\begin{array}{l}16(-) \\
16(-)\end{array}$ & $\begin{array}{l}27.6 \\
23.1 \\
\end{array}$ & $\begin{array}{l}19 \mathrm{~m} \\
24 \mathrm{y}\end{array}$ & $\mathrm{R}=\mathrm{L}$ & Dyspepsia \\
\hline $\begin{array}{l}115 \\
116\end{array}$ & S. F. & $\begin{array}{l}9(+) \\
9(+)\end{array}$ & $\begin{array}{l}20.0 \\
15.8 \\
\end{array}$ & $\begin{array}{l}10 \mathrm{~m} \\
27 \mathrm{y}\end{array}$ & $\mathrm{R}=\mathrm{L}_{\mathrm{p}}$ & Tracheobronchitis \\
\hline $\begin{array}{l}117 \\
118 \\
\end{array}$ & S. S. & $\begin{array}{r}11( \pm) \\
2(H)\end{array}$ & $\begin{array}{r}20.0 \\
8.6 \\
\end{array}$ & $\begin{array}{l}16 \mathrm{~m} \\
40 \mathrm{y}\end{array}$ & $\mathrm{R}=\mathrm{L}$ & Bronchopneumonia \\
\hline $\begin{array}{l}119 \\
120 \\
\end{array}$ & Y. K. & $\begin{array}{l}16(-) \\
16(-)\end{array}$ & $\begin{array}{l}75.0 \\
75.0 \\
\end{array}$ & $\begin{array}{r}2 \mathrm{~m} \\
38 \mathrm{y}\end{array}$ & $\mathrm{R}<\mathrm{L}$ & Osteochondritis syphilitica \\
\hline $\begin{array}{l}121 \\
122 \\
\end{array}$ & T. T. & $\begin{array}{l}14(-) \\
13(-)\end{array}$ & $\begin{array}{l}47.5 \\
35.4 \\
\end{array}$ & $\begin{array}{l}12 \mathrm{~m} \\
39 \mathrm{y}\end{array}$ & $\mathrm{R}=\mathrm{L}_{\mathrm{p}}$ & Bronchopneumonia + atrophy \\
\hline $\begin{array}{l}123 \\
124 \\
\end{array}$ & K. W. & $\begin{array}{l}1(H) \\
1(H)\end{array}$ & $\begin{array}{l}14.1 \\
13.0 \\
\end{array}$ & $\begin{array}{l}11 \mathrm{~m} \\
25 \mathrm{y}\end{array}$ & $\mathrm{R}=\mathrm{L}$ & Angina \\
\hline $\begin{array}{l}125 \\
126 \\
\end{array}$ & E. O. & $\begin{array}{l}2(+) \\
1(H)\end{array}$ & $\begin{array}{l}5.0 \\
5.0 \\
\end{array}$ & $\begin{array}{r}9 \mathrm{~m} \\
33 \mathrm{y} \\
\end{array}$ & $\mathrm{R}>\mathrm{L}_{\mathrm{p}}$ & Rhinopharyngitis \\
\hline $\begin{array}{l}127 \\
128 \\
\end{array}$ & S. S. & $\begin{array}{r}16(-) \\
7(-)\end{array}$ & $\begin{array}{l}66.6 \\
50.0 \\
\end{array}$ & $\begin{array}{c}16 \mathrm{~m} \\
*\end{array}$ & $\mathrm{R}<\mathrm{L}_{\mathrm{p}}$ & Severe dyspepsia \\
\hline $\begin{array}{l}129 \\
130 \\
\end{array}$ & K. A. & $\begin{array}{r}6( \pm) \\
12(-)\end{array}$ & $\begin{array}{l}11.5 \\
45.0 \\
\end{array}$ & $\begin{array}{r}7 \mathrm{~m} \\
26 \mathrm{y} \\
\end{array}$ & $\mathrm{R}>\mathrm{L}$ & B-avitaminotic dyspepsia \\
\hline $\begin{array}{l}131 \\
132\end{array}$ & E. 1 . & $\begin{array}{l}16(-) \\
16(-)\end{array}$ & $\begin{array}{l}40.0 \\
30.0 \\
\end{array}$ & $\begin{array}{r}2 \mathrm{~m} \\
24 \mathrm{y} \\
\end{array}$ & $\mathrm{R}=\mathrm{L}_{\mathrm{p}}$ & Healthy \\
\hline $\begin{array}{l}133 \\
134\end{array}$ & T. $\mathrm{H}$. & $\begin{array}{l}6(-) \\
1(H)\end{array}$ & $\begin{array}{l}28.5 \\
13.6\end{array}$ & $\begin{array}{l}10 \mathrm{~m} \\
23 \mathrm{y}\end{array}$ & $\mathrm{R}=\mathrm{L}$ & Infantile preberiberi \\
\hline
\end{tabular}


TABle

\begin{tabular}{|c|c|c|c|c|c|c|}
\hline No. & Name & $\begin{array}{l}\text { Ara- } \\
\text { kawa's } \\
\text { reaction }\end{array}$ & $\begin{array}{l}\text { Diastase } \\
\text { content }\end{array}$ & $\begin{array}{c}\text { Age of } \\
\text { children } \\
\mathrm{m}=\text { months } \\
\text { age of } \\
\text { mothers } \\
\mathrm{y}=\text { years }\end{array}$ & $\begin{array}{c}\text { Condition } \\
\text { of } \\
\text { secretion }\end{array}$ & Diagnosis \\
\hline $\begin{array}{l}135 \\
136 \\
\end{array}$ & S. O. & $\begin{array}{r}16(-) \\
5( \pm)\end{array}$ & $\begin{array}{l}6.7 \\
6.7 \\
\end{array}$ & $\begin{array}{r}9 \mathrm{~m} \\
28 \mathrm{y} \\
\end{array}$ & $\mathbf{R}=\mathbf{L}$ & Infantile preberiberi \\
\hline $\begin{array}{l}137 \\
138 \\
\end{array}$ & K. N. & $\begin{array}{r}6( \pm) \\
16(-)\end{array}$ & $\begin{array}{l}14.5 \\
12.8 \\
\end{array}$ & $\begin{array}{r}2 \mathrm{~m} \\
23 \mathrm{y} \\
\end{array}$ & $\mathrm{R}=\mathrm{L}_{\mathrm{p}}$ & Infantile preberiberi \\
\hline $\begin{array}{l}139 \\
140\end{array}$ & K. S. & $\begin{array}{l}1(H) \\
1(H)\end{array}$ & $\begin{array}{l}13.0 \\
13.0\end{array}$ & $\begin{array}{r}3 \mathrm{~m} \\
26 \mathrm{y} \\
\end{array}$ & $\mathrm{R}=\mathrm{L}$ & Human milk intoxication \\
\hline $\begin{array}{l}141 \\
142 \\
\end{array}$ & N. K. & $\begin{array}{l}16(-) \\
15(-)\end{array}$ & $\begin{array}{l}27.6 \\
25.0\end{array}$ & $\begin{array}{r}6 \mathrm{~m} \\
31 \mathrm{y} \\
\end{array}$ & $\mathrm{R}<\mathrm{L}$ & Infantile preberiberi \\
\hline $\begin{array}{l}143 \\
144\end{array}$ & K. K. & $\mid \begin{array}{c} \pm+H+H \\
16(-)\end{array}$ & $\begin{array}{l}13.0 \\
10.0\end{array}$ & $\begin{array}{c}19 \mathrm{~m} \\
*\end{array}$ & * & (T) \\
\hline \begin{tabular}{|l}
145 \\
146 \\
\end{tabular} & T. T. & $\begin{array}{l}16(-) \\
16(-)\end{array}$ & $\begin{array}{l}40.0 \\
37.5 \\
\end{array}$ & $\begin{array}{c}2 \mathrm{~m} \\
*\end{array}$ & $\mathbf{R}=\mathbf{L}$ & $\begin{array}{l}\text { Bronchopneumonia } \uparrow \text { infantile } \\
\text { preberiberi }\end{array}$ \\
\hline $\begin{array}{l}147 \\
148 \\
\end{array}$ & G. Y. & $\begin{array}{r}8(-) \\
14(-)\end{array}$ & $\begin{array}{l}4.5 \\
5.5 \\
\end{array}$ & $\begin{array}{l}3 \mathrm{~m} \\
*\end{array}$ & $\mathrm{R}=\mathrm{L}$ & Infantile beriberi \\
\hline $\begin{array}{l}149 \\
150\end{array}$ & K. K. & $\begin{array}{l}16(-) \\
16(-)\end{array}$ & $\begin{array}{l}23.1 \\
23.1\end{array}$ & $\begin{array}{r}1 \mathrm{~m} \\
21 \mathrm{y} \\
\end{array}$ & $\mathrm{R}=\mathrm{L}$ & Infantile preberiberi \\
\hline $\begin{array}{l}151 \\
152\end{array}$ & T. M. & $\begin{array}{r}16(-) \\
2(H)\end{array}$ & $\begin{array}{r}21.0 \\
8.3 \\
\end{array}$ & $\begin{array}{l}18 \mathrm{~m} \\
22 \mathrm{y} \\
\end{array}$ & $\mathrm{R}=\mathrm{L}$ & Dyspepsia \\
\hline $\begin{array}{l}153 \\
154\end{array}$ & S. T. & $\begin{array}{r}13( \pm) \\
2(+)\end{array}$ & $\begin{array}{l}28.5 \\
13.1 \\
\end{array}$ & $\begin{array}{l}15 \mathrm{~m} \\
28 \mathrm{y} \\
\end{array}$ & $\mathrm{R}=\mathrm{L}$ & Rhinopharyngitis \\
\hline $\begin{array}{l}155 \\
156\end{array}$ & H. S. & $\begin{array}{r}6(-) \\
11(-) \\
\end{array}$ & $\begin{array}{l}36.0 \\
37.5\end{array}$ & $\begin{array}{r}1 \mathrm{~m} \\
23 \mathrm{y} \\
\end{array}$ & $\mathrm{R}=\mathrm{L}$ & Infantile beriberi \\
\hline $\begin{array}{l}157 \\
158\end{array}$ & A. $K$. & $\begin{array}{l}3(-5) \\
5( \pm)\end{array}$ & $\begin{array}{l}7.5 \\
7.5 \\
\end{array}$ & $\begin{array}{l}18 \mathrm{~m} \\
28 \mathrm{y}\end{array}$ & $\mathrm{R}=\mathrm{L}$ & Dyspepsia \\
\hline $\begin{array}{l}159 \\
160 \\
\end{array}$ & Z.W. & $\begin{array}{r}11(-) \\
4(-)\end{array}$ & $\begin{array}{l}50.0 \\
42.8 \\
\end{array}$ & $\begin{array}{r}8 \mathrm{~m} \\
24 \mathrm{y} \\
\end{array}$ & $\mathrm{R}=\mathrm{L}$ & $\begin{array}{l}\text { Dyspepsia }+ \text { bronchopneumo- } \\
\text { nia }\end{array}$ \\
\hline $\begin{array}{l}161 \\
162 \\
\end{array}$ & T. O. & $\begin{array}{l}1(H) \\
1(H) \\
\end{array}$ & $\begin{array}{l}15.0 \\
13.6 \\
\end{array}$ & $\begin{array}{r}8 \mathrm{~m} \\
26 \mathrm{y} \\
\end{array}$ & $\mathrm{R}=\mathrm{L}$ & Dyspepsia \\
\hline $\begin{array}{l}163 \\
164 \\
\end{array}$ & Y. O. & $\begin{array}{l}1(H) \\
2(H) \\
\end{array}$ & $\begin{array}{l}27.6 \\
27.6 \\
\end{array}$ & $\begin{array}{r}9 \mathrm{~m} \\
24 \mathrm{y} \\
\end{array}$ & $\mathrm{R}>\mathrm{L}$ & Amaurosis + dyspepsia \\
\hline $\begin{array}{l}165 \\
166 \\
\end{array}$ & Y. H. & $\begin{array}{l}6(+) \\
2(H) \\
\end{array}$ & $\begin{array}{l}33.4 \\
29.1 \\
\end{array}$ & $\begin{array}{l}15 \mathrm{~m} \\
22 \mathrm{y}\end{array}$ & $\mathrm{R}<\mathrm{L}$ & Angina \\
\hline $\begin{array}{l}167 \\
168 \\
\end{array}$ & Y. S. & $\begin{array}{r}1(t) \\
15(t) \\
\end{array}$ & $\begin{array}{r}8.3 \\
13.6 \\
19\end{array}$ & $\begin{array}{l}10 \mathrm{~m} \\
33 \mathrm{y}\end{array}$ & $\mathrm{R}>\mathrm{L}$ & Acute dyspepsia \\
\hline $\begin{array}{l}169 \\
170 \\
\end{array}$ & N. K. & $\begin{array}{l}1(+) \\
5( \pm)\end{array}$ & $\begin{array}{l}14.2 \\
14.2 \\
14\end{array}$ & $\begin{array}{l}6 \mathrm{~m} \\
39 \mathrm{y}\end{array}$ & $\mathrm{R}>\mathrm{L}$ & Rhinopharyngitis \\
\hline $\begin{array}{l}171 \\
172 \\
\end{array}$ & E. $O$. & $\begin{array}{r}1(+) \\
14( \pm)\end{array}$ & $\begin{array}{l}11.1 \\
32.2 \\
\end{array}$ & $\begin{array}{r}4 \mathrm{~m} \\
35 \mathrm{y} \\
\end{array}$ & $\mathrm{R}>\mathrm{L}$ & Bronchopneumonia \\
\hline $\begin{array}{l}173 \\
174 \\
\end{array}$ & T. K. & $\begin{array}{r}12(-) \\
6(-) \\
\end{array}$ & $\begin{array}{l}9.4 \\
9.4 \\
\end{array}$ & $\begin{array}{c}4 \mathrm{~m} \\
23 \mathrm{y} \\
\end{array}$ & $\mathrm{R}>\mathrm{L}$ & B-avitaminotic dyspepsia \\
\hline $\begin{array}{l}175 \\
176 \\
\end{array}$ & Y. S. & $\begin{array}{r}16(-) \\
3(+) \\
\end{array}$ & $\begin{array}{r}9.4 \\
9.4 \\
10\end{array}$ & $\begin{array}{l}15 \mathrm{~m} \\
43 \mathrm{y}\end{array}$ & $\mathrm{R}=\mathrm{L}$ & Rhinopharyngitis \\
\hline $\begin{array}{l}177 \\
178 \\
\end{array}$ & S. S. & $\begin{array}{l}3(+) \\
5( \pm) \\
\end{array}$ & $\begin{array}{l}10.0 \\
10.0 \\
17\end{array}$ & $\begin{array}{c}15 \mathrm{~m} \\
* \\
\end{array}$ & $\mathrm{R}<\mathrm{L}$ & Rhinopharyngitis \\
\hline $\begin{array}{l}179 \\
180 \\
\end{array}$ & H. S. & $\begin{array}{r}10( \pm) \\
6(+)\end{array}$ & $\begin{array}{l}17.6 \\
14.8 \\
375\end{array}$ & $\begin{array}{c}7 \mathrm{~m} \\
*\end{array}$ & $\mathrm{R}<\mathrm{L}$ & Angina + bronchitis \\
\hline $\begin{array}{l}181 \\
182 \\
\end{array}$ & T. O. & $\begin{array}{l}16(-) \\
14( \pm)\end{array}$ & $\begin{array}{l}37.5 \\
27.6 \\
33.4\end{array}$ & $\begin{array}{l}1 \mathrm{~m} \\
27 \mathrm{y}\end{array}$ & $\mathrm{R}=\mathrm{L}_{\mathrm{p}}$ & Fever \\
\hline $\begin{array}{l}183 \\
184 \\
\end{array}$ & W. K. & $\begin{array}{l}15(-) \\
14(-)\end{array}$ & $\begin{array}{l}33.4 \\
27.6 \\
\end{array}$ & $\begin{array}{r}3 \mathrm{~m} \\
32 \mathrm{y} \\
\end{array}$ & $R=L$ & Rhinopharyngitis \\
\hline $\begin{array}{l}185 \\
186\end{array}$ & Z. N. & $\begin{array}{r}8(-) \\
11(+)\end{array}$ & $\begin{array}{l}21.5 \\
18.8\end{array}$ & $\begin{array}{l}10 \mathrm{~m} \\
25 \mathrm{y}\end{array}$ & $\mathrm{R}=\mathrm{L}$ & Acute dyspepsia \\
\hline
\end{tabular}


I. Cọntinued (3).

\begin{tabular}{|c|c|c|c|c|c|c|}
\hline No. & Name & $\begin{array}{l}\text { Ara- } \\
\text { kawa's } \\
\text { reaction }\end{array}$ & $\begin{array}{l}\text { Diastase } \\
\text { content }\end{array}$ & $\begin{array}{c}\text { Age of } \\
\text { children } \\
\mathrm{m}=\text { months } \\
\text { age of } \\
\text { mothers } \\
\mathrm{y}=\text { years }\end{array}$ & $\begin{array}{l}\text { Condition } \\
\text { of } \\
\text { secretion }\end{array}$ & Diagnosis \\
\hline $\begin{array}{l}187 \\
188\end{array}$ & A. $\mathbf{N}$. & $\begin{array}{l}1(H) \\
1(H)\end{array}$ & $\begin{array}{l}8.8 \\
8.8\end{array}$ & $\begin{array}{l}7 \mathrm{~m} \\
26 \mathrm{y}\end{array}$ & $\mathrm{R}>\mathrm{L}_{\mathrm{p}}$ & Anaemia $f$ laryngitis \\
\hline $\begin{array}{l}189 \\
190 \\
\end{array}$ & R. F. & $\begin{array}{l}1(H) \\
1(H)\end{array}$ & $\begin{array}{l}18.8 \\
20.0\end{array}$ & $\begin{array}{l}15 \mathrm{~m} \\
27 \mathrm{y}\end{array}$ & $\mathrm{R}=\mathrm{L}$ & Dyspepsia \\
\hline $\begin{array}{l}191 \\
192 \\
\end{array}$ & S. I. & $\begin{array}{r}6(-) \\
12(-) \\
\end{array}$ & $\begin{array}{l}16.6 \\
18.8 \\
\end{array}$ & $\begin{array}{r}1 \mathrm{~m} \\
25 \mathrm{y}\end{array}$ & $\mathrm{R}>\mathrm{L}$ & Dyspepsia \\
\hline $\begin{array}{l}193 \\
194 \\
\end{array}$ & S. S. & $\begin{array}{l}10( \pm) \\
12( \pm) \\
\end{array}$ & $\begin{array}{r}10.0 \\
-10.0 \\
\end{array}$ & $\begin{array}{r}6 \mathrm{~m} \\
37 \mathrm{y}\end{array}$ & $\mathrm{R}=\mathrm{L}$ & B-avitaminotic dystrophy \\
\hline $\begin{array}{l}195 \\
196\end{array}$ & S. O. & $\begin{array}{r}12(-) \\
4(+) \\
\end{array}$ & $\begin{array}{l}60.0 \\
20.0 \\
\end{array}$ & $\begin{array}{l}8 \mathrm{~m} \\
28 \mathrm{y}\end{array}$ & $\mathrm{R}<\mathrm{L}$ & Eczema $\dagger$ dyspepsia \\
\hline $\begin{array}{l}197 \\
198 \\
\end{array}$ & N. S. & $\begin{array}{l}12(-) \\
15(-)\end{array}$ & $\begin{array}{l}25.0 \\
27: 0\end{array}$ & $\begin{array}{l}13 \mathrm{~m} \\
32 \mathrm{y}\end{array}$ & $\mathrm{R}=\mathrm{L}$ & $\begin{array}{l}\text { Hereditary dislocation of } \\
\text { hipjoint }\end{array}$ \\
\hline $\begin{array}{l}199 \\
200\end{array}$ & E. $\mathbf{N}$. & $\begin{array}{l}4(H) \\
4(H)\end{array}$ & $\begin{array}{r}15.0 \\
8.8 \\
\end{array}$ & $\begin{array}{r}9 \mathrm{~m} \\
21 \mathrm{y}\end{array}$ & $\mathrm{R}=\mathrm{L}$ & Bronchitis \\
\hline $\begin{array}{l}201 \\
202 \\
\end{array}$ & Y. S. & $\begin{array}{l}1(\#) \\
1(H)\end{array}$ & $\begin{array}{l}15.0 \\
15.0\end{array}$ & $\begin{array}{l}20 \mathrm{~m} \\
32 \mathrm{y}^{\circ}\end{array}$ & $\mathrm{R}=\mathrm{L}$ & Impetigo \\
\hline $\begin{array}{l}203 \\
204\end{array}$ & T. S. & $\begin{array}{l}2( \pm) \\
1(+)\end{array}$ & $\begin{array}{l}21.5 \\
12.0\end{array}$ & $\begin{array}{l}9 \mathrm{~m} \\
33 \mathrm{y}\end{array}$ & $\mathrm{R}<\mathrm{L}$ & Rhinopharyngitis \\
\hline $\begin{array}{l}205 \\
206\end{array}$ & E. F. & $\begin{array}{r}12( \pm) \\
1(\#)\end{array}$ & $\begin{array}{r}27.6 \\
9.7 \\
\end{array}$ & $\begin{array}{r}3 \mathrm{~m} \\
23 \mathrm{y}\end{array}$ & $\mathrm{R}=\mathrm{L}$ & Dyspepsia \\
\hline $\begin{array}{l}207 \\
208\end{array}$ & H. Y. & $\left|\begin{array}{c} \pm+H H H \\
1(H)\end{array}\right|$ & $\begin{array}{l}5.0 \\
5.0\end{array}$ & $\begin{array}{l}6 \mathrm{~m} \\
26 \mathrm{y}\end{array}$ & $\mathrm{R}=\mathrm{L}$ & Dyspepsia \\
\hline $\begin{array}{l}209 \\
210 \\
\end{array}$ & S. A. & $\begin{array}{l}1(\mathrm{H}) \\
1(\mathrm{H}) \\
\end{array}$ & $\begin{array}{r}13.6 \\
7.5 \\
\end{array}$ & $\begin{array}{r}5 \mathrm{~m} \\
29 \mathrm{y} \\
\end{array}$ & $\mathrm{R}>\mathrm{L}$ & Dyspepsia \\
\hline $\begin{array}{l}211 \\
212\end{array}$ & K. I. & $\begin{array}{r}15(-) \\
8(-)\end{array}$ & $\begin{array}{l}5.0 \\
5.0\end{array}$ & $\begin{array}{c}4 \mathrm{~m} \\
22 \mathrm{y}\end{array}$ & $\mathrm{R}=\mathrm{L}$ & Dyspepsia \\
\hline $\begin{array}{l}13 \\
214 \\
\end{array}$ & M. M. & $\begin{array}{c} \pm \pm \#+\# \\
1(H)\end{array}$ & $\begin{array}{r}21.5 \\
8.1 \\
\end{array}$ & $\begin{array}{r}7 \mathrm{~m} \\
33 \mathrm{y}\end{array}$ & $\mathrm{R}=\mathrm{L}$ & Acute dyspepsia \\
\hline $\begin{array}{l}215 \\
216 \\
\end{array}$ & $\mathrm{~K}: \mathbf{S}$ & $\begin{array}{l}1(\mathrm{~m}) \\
1(\mathrm{~m}) \\
\end{array}$ & $\begin{array}{l}13.6 \\
16.6 \\
\end{array}$ & $\begin{array}{l}6 \mathrm{~m} \\
* \\
\end{array}$ & $\mathrm{R}=\mathrm{L}$ & Tracheobronchitis \\
\hline $\begin{array}{l}217 \\
218 \\
\end{array}$ & T. T. & $\begin{array}{l}1(H) \\
1(H)\end{array}$ & $\begin{array}{l}20.0 \\
14.2 \\
\end{array}$ & $\begin{array}{r}7 \mathrm{~m} \\
23 \mathrm{y} \\
\end{array}$ & $\mathrm{R}>\mathrm{L}$ & Dyspepsia \\
\hline $\begin{array}{l}219 \\
220\end{array}$ & S. A. & $\begin{array}{l}8(-) \\
6( \pm)\end{array}$ & $\begin{array}{l}16.6 \\
16.6\end{array}$ & $\begin{array}{l}16 \mathrm{~m} \\
21 \mathrm{y}\end{array}$ & $\mathrm{R}=\mathrm{L}$ & Dyspepsia \\
\hline $\begin{array}{l}221 \\
222\end{array}$ & K. A. & $\begin{array}{l}11( \pm) \\
11( \pm)\end{array}$ & $\begin{array}{l}15.0 \\
15.0\end{array}$ & $\begin{array}{c}15 \mathrm{~m} \\
*\end{array}$ & $\mathrm{R}=\mathrm{L}$ & Dystrophy \\
\hline $\begin{array}{l}223 \\
224 \\
\end{array}$ & $K, K$ & $\begin{array}{l}1(+) \\
1(+)\end{array}$ & $\begin{array}{l}17.6 \\
18.8 \\
\end{array}$ & $\begin{array}{r}5 \mathrm{~m} \\
23 \mathrm{y} \\
\end{array}$ & $\mathrm{R}=\mathrm{L}$ & Influenza \\
\hline $\begin{array}{l}225 \\
226 \\
\end{array}$ & I. K. & $\begin{array}{l}1(H) \\
1(H)\end{array}$ & $\begin{array}{l}8.3 \\
6.0 \\
\end{array}$ & $\begin{array}{c}10 \mathrm{~m} \\
*\end{array}$ & $\mathrm{R}=\mathrm{L}$ & * \\
\hline $\begin{array}{l}227 \\
228 \\
\end{array}$ & K. K. & $\left|\begin{array}{c} \pm \pm+H+H \\
16(-)\end{array}\right|$ & $\begin{array}{l}25.0 \\
16.6 \\
\end{array}$ & * & $\mathrm{R}>\mathrm{L}$ & * \\
\hline $\begin{array}{l}229 \\
230 \\
\end{array}$ & K. O. & \begin{tabular}{r|}
$7( \pm)$ \\
$11( \pm)$ \\
\end{tabular} & $\begin{array}{l}23.1 . \\
23.1\end{array}$ & $\begin{array}{c}19 \mathrm{~m} \\
*\end{array}$ & $\mathrm{R}=\mathrm{L}$ & Urticaria \\
\hline $\begin{array}{l}231 \\
232\end{array}$ & Y. O. & $\begin{array}{l}1(H) \\
1(H)\end{array}$ & $\begin{array}{l}12.2 \\
12.2 \\
\end{array}$ & $\begin{array}{l}18 \mathrm{~m} \\
33 \mathrm{y}\end{array}$ & $\mathrm{R}=\mathrm{L}$ & Pulmonary tuberculosis \\
\hline $\begin{array}{l}233 \\
234 \\
\end{array}$ & R. S. & $\begin{array}{r}13( \pm) \\
1(H)\end{array}$ & $\begin{array}{l}42.8 \\
12.2 \\
\end{array}$ & $\begin{array}{r}8 \mathrm{~m} \\
32 \mathrm{y} \\
\end{array}$ & $\mathrm{R}<\mathrm{L}$ & Rhinopharyngitis \\
\hline $\begin{array}{l}235 \\
236 \\
\end{array}$ & A. A. & $\begin{array}{l}16(-) \\
16(-)\end{array}$ & $\begin{array}{r}100.0 \\
75.0 \\
\end{array}$ & $\begin{array}{r}2 \mathrm{~m} \\
22 \mathrm{y}\end{array}$ & $\mathrm{R}=\mathrm{L}$ & Icterus neonatorum \\
\hline $\begin{array}{l}237 \\
238\end{array}$ & Y. M. & $\begin{array}{r}1(+4) \\
13( \pm)\end{array}$ & $\begin{array}{l}16.6 \\
18.8\end{array}$ & $\begin{array}{r}7 \mathrm{~m} \\
25 \mathrm{y}\end{array}$ & $\mathrm{R}=\mathrm{L}$ & Dyspepsia \\
\hline
\end{tabular}


TABLE

\begin{tabular}{|c|c|c|c|c|c|c|}
\hline No. & Name & $\begin{array}{l}\text { Ara- } \\
\text { kawa's } \\
\text { reaction }\end{array}$ & $\begin{array}{l}\text { Diastase } \\
\text { content }\end{array}$ & $\begin{array}{c}\text { Age of } \\
\text { children } \\
\mathrm{m}=\text { months } \\
\text { age of } \\
\text { mothers } \\
\mathrm{y}=\text { years }\end{array}$ & $\begin{array}{c}\text { Condition } \\
\text { of } \\
\text { secretion }\end{array}$ & Diagnosis \\
\hline $\begin{array}{l}239 \\
240 \\
\end{array}$ & K. W. & $\begin{array}{l}1(t+) \\
1(t+)\end{array}$ & $\begin{array}{l}8.3 \\
6.8 \\
\end{array}$ & $\begin{array}{c}9 \mathrm{~m} \\
27 \mathrm{y}\end{array}$ & $\mathrm{R}=\mathbf{L}$ & Dyspepsia \\
\hline $\begin{array}{l}241 \\
242\end{array}$ & C. $\mathrm{O}$. & $\begin{array}{l}1( \pm) \\
2(+)\end{array}$ & $\begin{array}{r}8.8 \\
12.2\end{array}$ & $\begin{array}{l}6 \mathrm{~m} \\
*\end{array}$ & $\mathbf{R}=\mathbf{L}$ & Healthy \\
\hline \begin{tabular}{|l}
243 \\
244
\end{tabular} & A. I. & $\left|\begin{array}{l}+H+H H+H \\
+H+H+H\end{array}\right|$ & $\begin{array}{l}23.1 \\
23.1 \\
\end{array}$ & $\begin{array}{l}10 \mathrm{~m} \\
28 \mathrm{y}\end{array}$ & $\mathbf{R}=\mathbf{L}$ & Strophulus \\
\hline $\begin{array}{l}245 \\
246\end{array}$ & Y. A. & $\begin{array}{c}16(-) \\
8(-)\end{array}$ & $\begin{array}{l}28.6 \\
28.6 \\
\end{array}$ & $\begin{array}{r}1 \mathrm{~m} \\
27 \mathrm{y} \\
\end{array}$ & $\mathbf{R}=\mathbf{L}$ & Dyspepsia \\
\hline $\begin{array}{l}247 \\
248 \\
\end{array}$ & K. Y. & $\begin{array}{l}1(\mathrm{H}) \\
1(\mathrm{H})\end{array}$ & $\begin{array}{l}13.6 \\
13.6 \\
\end{array}$ & $\begin{array}{r}5 \mathrm{~m} \\
34 \mathrm{y} \\
\end{array}$ & $\mathrm{R}=\mathrm{L}$ & Dyspepsia \\
\hline $\begin{array}{l}249 \\
250 \\
\end{array}$ & K. N. & $\begin{array}{l}16(-) \\
16(-)\end{array}$ & $\begin{array}{l}37.5 \\
27.6 \\
\end{array}$ & $\begin{array}{r}3 \mathrm{~m} \\
31 \mathrm{y} \\
\end{array}$ & $\mathrm{R}<\mathrm{L}$ & Dyspepsia \\
\hline $\begin{array}{l}251 \\
252 \\
\end{array}$ & I. $\mathrm{K}$. & $\begin{array}{l}1(H) \\
1(H)\end{array}$ & $\begin{array}{l}15.8 \\
15.8 \\
\end{array}$ & $\begin{array}{l}9 \mathrm{~m} \\
*\end{array}$ & $\mathrm{R}=\mathrm{L}$ & Acute dyspepsia \\
\hline $\begin{array}{l}253 \\
254\end{array}$ & X.S. & $\begin{array}{r}1(t+1) \\
15( \pm)\end{array}$ & $\begin{array}{l}15.0 \\
15.0 \\
\end{array}$ & $\begin{array}{c}9 \mathrm{~m} \\
* \\
\end{array}$ & $\mathrm{R}=\mathrm{L}$ & Acute dyspepsia \\
\hline $\begin{array}{l}255 \\
256\end{array}$ & S. M. & $\begin{array}{l}3(+) \\
1(t+)\end{array}$ & \begin{tabular}{|l}
16.6 \\
15.8 \\
\end{tabular} & $\begin{array}{c}5 \mathrm{~m} \\
*\end{array}$ & $\mathbf{R}=\mathbf{L}$ & Dyspepsia \\
\hline $\begin{array}{l}257 \\
258 \\
\end{array}$ & Y. S. & $\begin{array}{l}6( \pm) \\
7( \pm) \\
\end{array}$ & $\begin{array}{l}46.1 \\
46.1 \\
\end{array}$ & $\begin{array}{l}16 \mathrm{~m} \\
26 \mathrm{y} \\
\end{array}$ & $\mathbf{R}=\mathbf{L}$ & B-avitaminotic dyspepsia \\
\hline $\begin{array}{l}259 \\
260 \\
\end{array}$ & T. K. & $\begin{array}{l}1(\#) \\
1(H)\end{array}$ & $\begin{array}{l}37.5 \\
45.0 \\
\end{array}$ & $\begin{array}{l}13 \mathrm{~m} \\
27 \mathrm{y}\end{array}$ & $\mathbf{R}=\mathbf{L}$ & Kidney tumor \\
\hline $\begin{array}{l}261 \\
262 \\
\end{array}$ & T. O. & $\begin{array}{l}9(+) \\
3(H)\end{array}$ & $\begin{array}{l}17.0 \\
10.0 \\
\end{array}$ & $\begin{array}{r}6 \mathrm{~m} \\
27 \mathrm{y} \\
\end{array}$ & $\mathrm{R}<\mathrm{L}$ & Pharyngitis \\
\hline \begin{tabular}{|l}
263 \\
264 \\
\end{tabular} & F. Y. & $\begin{array}{r}13( \pm) \\
2(+)\end{array}$ & $\begin{array}{l}17.6 \\
25.0\end{array}$ & $\begin{array}{l}22 \mathrm{~m} \\
28 \mathrm{y}\end{array}$ & $\mathrm{R}<\mathrm{L}$ & Poliomyelitis anterior acuta \\
\hline $\begin{array}{l}265 \\
266\end{array}$ & M. O. & $\begin{array}{l}1(H) \\
1(H)\end{array}$ & $\begin{array}{l}8.3 \\
6.8 \\
\end{array}$ & $\begin{array}{r}8 \mathrm{~m} \\
25 \mathrm{y} .\end{array}$ & $\mathrm{R}<\mathrm{L}$ & Healthy \\
\hline $\begin{array}{l}267 \\
268 \\
\end{array}$ & H. C. & $\begin{array}{l}16(-) \\
16(-)\end{array}$ & $\begin{array}{l}36.1 \\
36.1 \\
\end{array}$ & $1 \mathrm{~m}$ & $\mathrm{R}=\mathrm{L}$ & $\begin{array}{l}\text { Ikterus neonatorum t heredi- } \\
\text { tary syphilis }\end{array}$ \\
\hline $\begin{array}{l}269 \\
270 \\
\end{array}$ & Y. O. & $\begin{array}{l}4(-) \\
1(H) \\
\end{array}$ & $\begin{array}{r}33.4 \\
9.5 \\
\end{array}$ & $\begin{array}{l}10 \mathrm{~m} \\
26 \mathrm{y}\end{array}$ & $\mathrm{R}=\mathrm{L}$ & Dyspepsia \\
\hline $\begin{array}{l}271 \\
272\end{array}$ & H. I. & $\begin{array}{l}1(+) \\
3(+)\end{array}$ & $\begin{array}{r}16.6 \\
23.1 \\
\end{array}$ & $\begin{array}{r}5 \mathrm{~m} \\
24 \mathrm{y}\end{array}$ & $\mathrm{R}=\mathrm{L}$ & Dyspepsia \\
\hline $\begin{array}{l}273 \\
274 \\
\end{array}$ & K. S. & $\begin{array}{l}2( \pm) \\
1(+)\end{array}$ & $\begin{array}{r}23.7 \\
16.6 \\
\end{array}$ & $\begin{array}{r}6 \mathrm{~m} \\
31 \mathrm{y} \\
\end{array}$ & $\mathrm{R}<\mathrm{L}$ & Dyspepsia \\
\hline $\begin{array}{l}275 \\
276 \\
\end{array}$ & X. K. & $\begin{array}{l}1(H) \\
1(H)\end{array}$ & $\begin{array}{r}8.8 \\
8.3 \\
\end{array}$ & $\begin{array}{r}8 \mathrm{~m} \\
26 \mathrm{y} \\
\end{array}$ & $\mathrm{R}=\mathrm{L}$ & Healthy \\
\hline $\begin{array}{l}277 \\
278 \\
\end{array}$ & $\mathrm{X} . \mathrm{K}$. & $\begin{array}{l}1(H) \\
1(H)\end{array}$ & $\begin{array}{l}10.0 \\
10.0\end{array}$ & $\begin{array}{r}6 \mathrm{~m} \\
26 \mathrm{y}\end{array}$ & $\mathrm{R}=\mathrm{L}$ & Healthy \\
\hline $\begin{array}{l}279 \\
280 \\
\end{array}$ & K. K. & $\begin{array}{l}6(-) \\
6(-)\end{array}$ & $\begin{array}{l}28.1 \\
26.5 \\
\end{array}$ & $\begin{array}{c}1 \mathrm{~m} \\
37 \mathrm{y}\end{array}$ & $\mathrm{R}=\mathrm{L}$ & Healthy \\
\hline $\begin{array}{l}281 \\
282 \\
\end{array}$ & X. T. & $\begin{array}{c}16(-) \\
6(-)\end{array}$ & $\begin{array}{r}150.0 \\
25.0 \\
\end{array}$ & $1_{*}^{1 \mathrm{~m}}$ & $\mathrm{R}=\mathrm{L}$ & * \\
\hline $\begin{array}{l}283 \\
284 \\
\end{array}$ & K. Y. & $\begin{array}{c}-+t++1 \\
6(-)\end{array}$ & $\begin{array}{l}25.0 \\
23.1 \\
\end{array}$ & $\begin{array}{c}2 \mathrm{~m} \\
*\end{array}$ & $*$ & Dyspepsia \\
\hline $\begin{array}{l}285 \\
286\end{array}$ & I. K. & $\begin{array}{r}8( \pm) \\
12(-)\end{array}$ & $\begin{array}{l}23.1 \\
20.0\end{array}$ & $\begin{array}{l}3 \mathrm{~m} \\
*\end{array}$ & $\mathrm{R}=\mathrm{L}$ & * \\
\hline $\begin{array}{l}287 \\
288 \\
\end{array}$ & Y. C. & $\begin{array}{r}16(-) \\
3( \pm) \\
\end{array}$ & $\begin{array}{r}20.0 \\
7.9 \\
176\end{array}$ & $\begin{array}{l}8 \mathrm{~m} \\
* \\
7 \mathrm{~m}\end{array}$ & $\mathrm{R}<\mathrm{L}$ & $*$ \\
\hline $\begin{array}{l}289 \\
290\end{array}$ & E. C. & $\begin{array}{l}2(+) \\
9( \pm)\end{array}$ & $\begin{array}{l}17.6 \\
10.0\end{array}$ & $7 \mathrm{~m}$ & $\mathrm{R}=\mathrm{L}$ & * \\
\hline
\end{tabular}


I. Continued (4).

\begin{tabular}{|c|c|c|c|c|c|c|}
\hline No. & Name & $\begin{array}{l}\text { Ara- } \\
\text { kawa's } \\
\text { reaction }\end{array}$ & $\begin{array}{c}\text { Diastase } \\
\text { content }\end{array}$ & $\begin{array}{l}\text { Age of } \\
\text { children } \\
\mathrm{m}=\text { months } \\
\text { age of } \\
\text { mothers } \\
\mathrm{y}=\text { years }\end{array}$ & $\begin{array}{l}\text { Condition } \\
\text { of } \\
\text { secretion }\end{array}$ & Diagnosis \\
\hline $\begin{array}{l}291 \\
292 \\
\end{array}$ & X. 0 . & $\begin{array}{l}15(-) \\
14(-)\end{array}$ & $\begin{array}{l}12.2 \\
12.2\end{array}$ & $\begin{array}{c}5 \mathrm{~m} \\
*\end{array}$ & $\mathrm{R}<\mathrm{L}_{\mathrm{p}}$ & Healthy \\
\hline $\begin{array}{l}293 \\
294\end{array}$ & R. A. & $\begin{array}{l}16(-) \\
16(-)\end{array}$ & $\begin{array}{l}54.2 \\
60.0\end{array}$ & $\begin{array}{r}3 \mathrm{~m} \\
25 \mathrm{y}\end{array}$ & $R=L$ & * \\
\hline $\begin{array}{l}295 \\
296 \\
\end{array}$ & K. I. & $\begin{array}{l}1(+) \\
4(+)\end{array}$ & $\begin{array}{l}23.1 \\
20.0\end{array}$ & $17 \mathrm{~m}$ & $\mathbf{R}<\mathrm{L}$ & Acute dyspepsia \\
\hline $\begin{array}{l}297 \\
298 \\
\end{array}$ & T. N. & $\begin{array}{l}1(H) \\
1(H)\end{array}$ & $\begin{array}{l}20.0 \\
18.4\end{array}$ & $\begin{array}{l}5 \mathrm{~m} \\
*\end{array}$ & $\mathbf{R}=\mathbf{L}$ & Healthy \\
\hline $\begin{array}{l}299 \\
300\end{array}$ & X. S. & $\begin{array}{l}1(\pi) \\
3( \pm)\end{array}$ & $\begin{array}{l}15.0 \\
25.0\end{array}$ & $14 \mathrm{~m}$ & $\mathrm{R}=\mathrm{L}$ & Pyothoracis \\
\hline $\begin{array}{l}301 \\
302\end{array}$ & K. O. & $\begin{array}{r}6(-) \\
16(-)\end{array}$ & $\begin{array}{l}33.4 \\
42.8 \\
\end{array}$ & $2 \mathrm{~m}$ & $\mathrm{R}=\mathrm{L}$ & Convulsion \\
\hline $\begin{array}{l}303 \\
304 \\
\end{array}$ & T. $\mathrm{T}$. & $\begin{array}{l}1(H) \\
1(\mathrm{H})\end{array}$ & $\begin{array}{r}10.7 \\
7.9 \\
\end{array}$ & $7 \mathrm{~m}$ & $\mathrm{R}>\mathrm{L}$ & Dyspepsia \\
\hline $\begin{array}{l}305 \\
306 \\
\end{array}$ & R. Y. & $\begin{array}{r}13( \pm) \\
4(+) \\
\end{array}$ & $\begin{array}{l}16.6 \\
10.7 \\
\end{array}$ & $\begin{array}{c}4 \mathrm{~m} \\
*\end{array}$ & $\mathrm{R}=\mathrm{L}$ & Dyspepsia \\
\hline $\begin{array}{l}307 \\
308 \\
\end{array}$ & T. K. & $\begin{array}{l}1(+1) \\
5(+)\end{array}$ & $\begin{array}{l}16.6 \\
27.6 \\
\end{array}$ & $\begin{array}{l}4 \mathrm{~m} \\
*\end{array}$ & $\mathrm{R}=\mathrm{L}$ & Measles \\
\hline $\begin{array}{l}309 \\
310\end{array}$ & T. N. & $\begin{array}{l}1(H) \\
1(t)\end{array}$ & $\begin{array}{l}23.1 \\
23.1\end{array}$ & ${ }_{*}^{2 \mathrm{~m}}$ & $\mathrm{R}=\mathrm{L}$ & Hernia ing. \\
\hline $\begin{array}{l}311 \\
312 \\
\end{array}$ & K. S. & $\begin{array}{l}1(\#) \\
1(H)\end{array}$ & $\begin{array}{l}7.1 \\
7.1 \\
\end{array}$ & $\begin{array}{c}5 \mathrm{~m} \\
*\end{array}$ & $*$ & Influenza \\
\hline $\begin{array}{l}313 \\
314\end{array}$ & M: Y. & $\begin{array}{l}1(+) \\
1(+)\end{array}$ & $\begin{array}{l}7.9 \\
9.4\end{array}$ & $\begin{array}{l}4 \mathrm{~m} \\
*\end{array}$ & $\mathrm{R}=\mathrm{L}$ & Dyspepsia \\
\hline $\begin{array}{l}315 \\
316\end{array}$ & Y. O. & $\begin{array}{l}7(+) \\
5( \pm)\end{array}$ & $\begin{array}{l}18.8 \\
20.0\end{array}$ & $2 \mathrm{~m}$ & $\mathrm{R}=\mathrm{L}$ & Infantile beriberi \\
\hline $\begin{array}{l}317 \\
318\end{array}$ & K. S. & $\begin{array}{r}6(-) \\
14(-)\end{array}$ & $\begin{array}{l}11.5 \\
13.6 \\
\end{array}$ & $2 \mathrm{~m}$ & $\mathrm{R}=\mathrm{G}$ & Influenza \\
\hline $\begin{array}{l}319 \\
320\end{array}$ & T. O. & $\begin{array}{l}10( \pm) \\
11( \pm)\end{array}$ & $\begin{array}{l}23.1 \\
21.5 \\
\end{array}$ & $10 \mathrm{~m}$ & $\mathbf{R}=\mathbf{L}$ & Infantile preberiberi \\
\hline $\begin{array}{l}321 \\
322\end{array}$ & T. O. & $\begin{array}{l}12(-) \\
16(-)\end{array}$ & $\begin{array}{l}30.0 \\
33.4 \\
\end{array}$ & $\begin{array}{l}2 \mathrm{~m} \\
*\end{array}$ & $\mathrm{R}>\mathrm{L}$ & Atrophy \\
\hline $\begin{array}{l}323 \\
324 \\
\end{array}$ & M. K. & $\begin{array}{r}12(-) \\
6(-)\end{array}$ & $\begin{array}{l}18.8 \\
18.8 \\
\end{array}$ & $3 \mathrm{~m}$ & $\mathrm{R}=\mathrm{L}$ & Rhinopharyngitis \\
\hline $\begin{array}{l}325 \\
326 \\
\end{array}$ & Y. S. & $\begin{array}{r}12(-) \\
6(-) \\
\end{array}$ & $\begin{array}{l}28.5 \\
27.6 \\
\end{array}$ & $\begin{array}{l}2 \mathrm{~m} \\
*\end{array}$ & $\mathrm{R}=\mathrm{L}$ & Obstipation \\
\hline $\begin{array}{l}327 \\
328 \\
\end{array}$ & K. S. & $\begin{array}{l}16(-) \\
16(-)\end{array}$ & $\begin{array}{l}28.5 \\
27.6 \\
\end{array}$ & $\begin{array}{c}1 \mathrm{~m} \\
*\end{array}$ & $\mathrm{R}=\mathrm{L}$ & Dystrophy \\
\hline $\begin{array}{l}329 \\
330\end{array}$ & Y. O. & $\begin{array}{l}1(H) \\
1(H)\end{array}$ & $\begin{array}{l}10.7 \\
10.7 \\
\end{array}$ & $\begin{array}{c}12 \mathrm{~m} \\
*\end{array}$ & $\mathrm{R}=\mathrm{L}$ & Angina \\
\hline $\begin{array}{l}331 \\
332 \\
\end{array}$ & M. K. & $\begin{array}{l}1(+1) \\
2(+)\end{array}$ & $\begin{array}{l}18.8 \\
20.0 \\
\end{array}$ & $\begin{array}{c}10 \mathrm{~m} \\
*\end{array}$ & $\mathrm{R}>\mathrm{L}$ & Dyspepsia \\
\hline $\begin{array}{l}333 \\
334 \\
\end{array}$ & K. S. & $\begin{array}{r}16(-) \\
3(+)\end{array}$ & $\begin{array}{l}50.0 \\
18.8 \\
\end{array}$ & $\begin{array}{l}5 \mathrm{~m} \\
*\end{array}$ & $\mathrm{R}<\mathrm{L}$ & Rhinopharyngitis \\
\hline $\begin{array}{l}335 \\
336 \\
\end{array}$ & M. I. & $\begin{array}{l}10( \pm) \\
16(-)\end{array}$ & $\begin{array}{l}42.8 \\
50.0 \\
\end{array}$ & $1 \mathrm{~m}$ & $\mathrm{R}<\mathrm{L}$ & Bronchopneumonia \\
\hline $\begin{array}{l}337 \\
338 \\
\end{array}$ & H. N. & $\begin{array}{l}1(H) \\
3(+)\end{array}$ & $\begin{array}{l}17.6 \\
18.8 \\
\end{array}$ & $20 \mathrm{~m}$ & $\mathrm{R}=\mathrm{L}$ & Rhinopharyngitis \\
\hline $\begin{array}{l}339 \\
340 \\
\end{array}$ & H. $O$. & $\begin{array}{l}7( \pm) \\
4(+) \\
\end{array}$ & $\begin{array}{l}25.0 \\
15.8 \\
\end{array}$ & $\begin{array}{l}6 \mathrm{~m} \\
*\end{array}$ & $\mathrm{R}=\mathrm{L}$ & Bronchitis \\
\hline $\begin{array}{l}341 \\
342\end{array}$ & K. Y. & $\begin{array}{l}1(H) \\
1(H)\end{array}$ & $\begin{array}{l}13.6 \\
13.6\end{array}$ & ${ }_{*}^{12 \mathrm{~m}}$ & $\mathrm{R}=\mathrm{L}$ & Angina \\
\hline
\end{tabular}


Table I. Continued (5).

\begin{tabular}{|c|c|c|c|c|c|c|}
\hline No. & Name & $\begin{array}{l}\text { Ara- } \\
\text { kawa's } \\
\text { reaction }\end{array}$ & $\begin{array}{l}\text { Diastase } \\
\text { content }\end{array}$ & $\begin{array}{c}\text { Age of } \\
\text { children } \\
\mathrm{m}=\text { months } \\
\text { age of } \\
\text { mothers } \\
\mathrm{y}=\text { years }\end{array}$ & $\begin{array}{c}\text { Condition } \\
\text { of } \\
\text { secretion }\end{array}$ & Diagnosis \\
\hline $\begin{array}{l}343 \\
344 \\
\end{array}$ & K. S. & $\begin{array}{r}16(-) \\
3(H)\end{array}$ & $\begin{array}{l}18.8 \\
18.8 \\
\end{array}$ & $\begin{array}{l}5 \mathrm{~m} \\
* \\
\end{array}$ & $\mathbf{R}<\mathbf{L}$ & Infantile preberiberi \\
\hline $\begin{array}{l}345 \\
346 \\
\end{array}$ & C. M. & $\begin{array}{l}16(-) \\
13(-)\end{array}$ & $\begin{array}{l}25.0 \\
24.4 \\
\end{array}$ & $\begin{array}{l}9 \mathrm{~m} \\
* \\
\end{array}$ & $\mathrm{R}=\mathrm{L}$ & Bronchitis \\
\hline $\begin{array}{l}347 \\
348 \\
\end{array}$ & K. T. & $\begin{array}{l}1(H) \\
1(H)\end{array}$ & $\begin{array}{l}11.5 \\
11.5 \\
\end{array}$ & $\begin{array}{l}4 \mathrm{~m} \\
*\end{array}$ & $\mathrm{R}=\mathrm{L}$ & Obstipation \\
\hline $\begin{array}{l}349 \\
350 \\
\end{array}$ & $Y: K$ & $\begin{array}{r}11(-) \\
5( \pm)\end{array}$ & $\begin{array}{l}21.5 \\
18.8 \\
\end{array}$ & $\begin{array}{l}9 \mathrm{~m} \\
*\end{array}$ & $\mathrm{R}>\mathrm{L}$ & Rhinopharyngitis \\
\hline $\begin{array}{l}351 \\
352\end{array}$ & F. T. & $\begin{array}{r}15(-) \\
5( \pm)\end{array}$ & $\begin{array}{r}100.0 \\
60.0 \\
\end{array}$ & $1 \mathrm{~m}$ & $\mathrm{R}>\mathrm{L}$ & Paresis of arm \\
\hline $\begin{array}{l}353 \\
354 \\
\end{array}$ & $\mathrm{~T} . \mathrm{K}$. & $\begin{array}{l}1(+) \\
5( \pm) \\
\end{array}$ & $\begin{array}{l}21.5 \\
23.1 \\
\end{array}$ & $\begin{array}{l}3 \mathrm{~m} \\
*\end{array}$ & $R=L$ & Influenza \\
\hline $\begin{array}{l}355 \\
356 \\
\end{array}$ & K. M. & $\begin{array}{l}4(-) \\
5( \pm) \\
\end{array}$ & $\begin{array}{l}21.5 \\
18.8 \\
\end{array}$ & $2 \mathrm{~m}$ & $\mathrm{R}=\mathrm{L}$ & Hereditary syphilis \\
\hline $\begin{array}{l}357 \\
358 \\
\end{array}$ & H. S. & $\begin{array}{l}7(+) \\
7(+)\end{array}$ & $\begin{array}{l}15.0 \\
15.0 \\
\end{array}$ & $\begin{array}{l}3 \mathrm{~m} \\
*\end{array}$ & $\mathrm{R}=\mathrm{L}$ & Atrophy \\
\hline $\begin{array}{l}359 \\
360 \\
\end{array}$ & $\mathrm{~T} . \mathrm{T}$ & $\begin{array}{l}1(+t) \\
5(t)\end{array}$ & $\begin{array}{l}12.2 \\
13.6 \\
\end{array}$ & $\begin{array}{l}4 \mathrm{~m} \\
*\end{array}$ & $\mathrm{R}=\mathrm{L}$ & Infantile preberiberi \\
\hline $\begin{array}{l}361 \\
362 \\
\end{array}$ & J. K. & $\begin{array}{l}1(H) \\
2( \pm)\end{array}$ & $\begin{array}{l}10.7 \\
25.0 \\
\end{array}$ & $\begin{array}{l}4 \mathrm{~m} \\
*\end{array}$ & $\mathrm{R}>\mathrm{L}$ & Dyspepsia \\
\hline $\begin{array}{l}363 \\
364 \\
\end{array}$ & K. Y. & $\begin{array}{l}1(+t) \\
3(+t)\end{array}$ & $\begin{array}{l}10.7 \\
11.5 \\
\end{array}$ & $\begin{array}{l}2 \mathrm{~m} \\
*\end{array}$ & * & Dyspepsia \\
\hline $\begin{array}{l}365 \\
366\end{array}$ & M. H. & $\begin{array}{l}2( \pm) \\
2( \pm)\end{array}$ & $\begin{array}{l}16.6 \\
13.6 \\
\end{array}$ & $\begin{array}{l}2 \mathrm{~m} \\
*\end{array}$ & $\mathrm{R}=\mathbf{L}$ & Nervosity \\
\hline $\begin{array}{l}367 \\
368\end{array}$ & H. O. & $\begin{array}{l}1(+) \\
1(+)\end{array}$ & $\begin{array}{l}21.5 \\
18.8\end{array}$ & $\begin{array}{l}5 \mathrm{~m} \\
*\end{array}$ & $\mathrm{R}=\mathrm{L}$ & Dyspepsia \\
\hline $\begin{array}{l}369 \\
370 \\
\end{array}$ & S. T. & $\begin{array}{r}6(t) \\
11( \pm) \\
\end{array}$ & $\begin{array}{l}25.0 \\
27.6 \\
\end{array}$ & $\begin{array}{l}6 \mathrm{~m} \\
*\end{array}$ & $\mathrm{R}=\mathrm{L}$ & Strophulus \\
\hline $\begin{array}{l}371 \\
372 \\
\end{array}$ & A. I. & $\begin{array}{l}1(+) \\
1(+)\end{array}$ & $\begin{array}{l}13.6 \\
15.0 \\
\end{array}$ & $\begin{array}{l}9 \mathrm{~m} \\
*\end{array}$ & $\mathrm{R}<\mathrm{L}$ & Eczema \\
\hline $\begin{array}{l}373 \\
374 \\
\end{array}$ & K. S. & $\begin{array}{c}1(+) \\
-+H+H\end{array}$ & $\begin{array}{l}12.2 \\
21.5\end{array}$ & $\begin{array}{l}3 \mathrm{~m} \\
*\end{array}$ & $\mathrm{R}>\mathrm{L}$ & Healthy \\
\hline $\begin{array}{l}375 \\
376 \\
\end{array}$ & T. I. & $\begin{array}{l}1(H) \\
1(H)\end{array}$ & $\begin{array}{l}18.8 \\
21.5 \\
\end{array}$ & $\begin{array}{l}4 \mathrm{~m} \\
*\end{array}$ & $\mathrm{R}<\mathrm{L}$ & Rhinopharyngitis \\
\hline $\begin{array}{l}377 \\
378 \\
\end{array}$ & K. N. & $\begin{array}{l}4(+) \\
7( \pm)\end{array}$ & $\begin{array}{l}10.7 \\
21.5 \\
\end{array}$ & * & $\mathrm{R}=\mathrm{L}$ & Rhinopharyngitis \\
\hline 379 & H. H. & $10(-)$ & $\begin{array}{c}60.0 \\
*\end{array}$ & $\begin{array}{l}22 \mathrm{~m} \\
40 \mathrm{y}\end{array}$ & $*$ & Postencephalitic paresis \\
\hline 380 & T. A. & $\begin{array}{c}* \\
1(\text { (\#) }\end{array}$ & $\begin{array}{c}* \\
27.6\end{array}$ & $\begin{array}{l}18 \mathrm{~m} \\
38 \mathrm{y}\end{array}$ & $\mathrm{R}<\mathrm{L}_{\mathrm{P}}$ & Pharyngitis \\
\hline
\end{tabular}

Table of signs for showing different A rakawa's reaction.

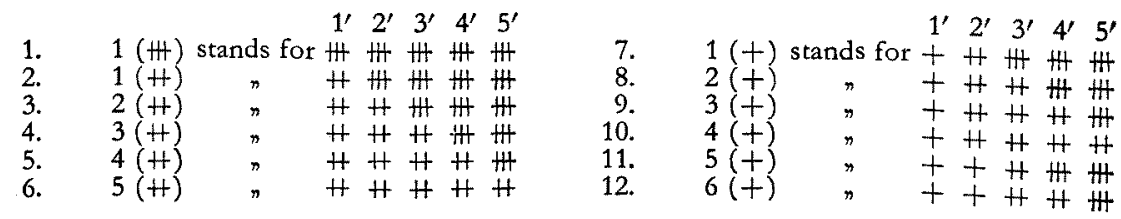


$1^{\prime} 2^{\prime} 3^{\prime} 4^{\prime} 5^{\prime}$

13. $7(+)$ stands for $++H+H$

15. $\quad 8(+) \Rightarrow+++H$

16. $10(+) \pi++++H$

17. $11(+)=\pi++++$

18. $1( \pm) \Rightarrow \pm+H$ \#

19. 2 (土) $" \pm+\#+\#$

20. $3( \pm) \quad \pm+H+H$

21. $4( \pm) \quad n \quad \pm++H$

22. $6( \pm) \quad \pm++H+$

23. $6( \pm)$

24. $7( \pm)$

25. $8( \pm)$

26. $9( \pm)$

27. $10( \pm)$

28. $11( \pm)$

29. $12( \pm)$

30. $13( \pm)$
$\pm+t+H$

$\pm+t+t$

$\pm \pm+H$ H

$\pm \pm+H+H$

$\pm \pm++H$

$\pm \pm+++$

$\pm \pm \pm+H$

$\pm \pm \pm++$

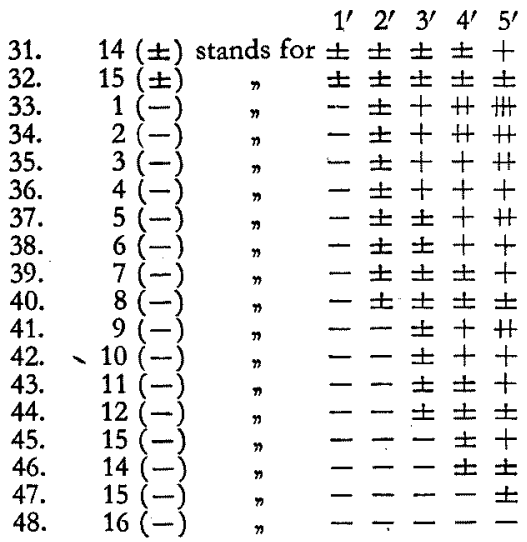

Explanation to the table:-

Take, for instance, the sign: $2(H)$. This stands for Arakawa's reaction with the course $(H) 1^{\prime}(H) 2^{\prime}(\#) 3^{\prime}(H) 4^{\prime}(H) 5^{\prime}$. The sign does not express any prompt result of the reaction, so the prompt reaction of the sign: $2^{\prime}(+)$ may be $(-) 0^{\prime},( \pm) 0^{\prime},(+) 0^{\prime}$ or even $(H) 0^{\prime}$, but this will not matter much, as the result of the reaction in one minute is the most important.

TABLE II.

Diastase content according to the intensity of A rakawa's reaction.

\begin{tabular}{|c|c|c|c|c|c|c|c|}
\hline \multirow{2}{*}{ Diastase } & \multirow{2}{*}{$\begin{array}{c}\text { All cases } \\
380 \text { cases } \\
\text { in all }\end{array}$} & \multicolumn{2}{|c|}{$\begin{array}{l}\text { Strongly Arakawa- } \\
\text { positive milk }\end{array}$} & \multicolumn{3}{|c|}{ Weakly Arakawa-positive milk } & \multirow{2}{*}{\begin{tabular}{|c} 
Completely \\
Arakawa- \\
negative milk \\
$\begin{array}{c}\text { A.R. }(-) 5^{\prime} \\
46 \text { cases }\end{array}$ \\
\end{tabular}} \\
\hline & & $\begin{array}{c}\text { A.R. }(H) 1 \\
55 \text { cases }\end{array}$ & $\begin{array}{c}\text { A.R. }(H) 1 \\
82 \text { cases }\end{array}$ & $\begin{array}{c}\text { A.R. }(+) 1 \\
63 \text { cases }\end{array}$ & $\begin{array}{l}\text { A.R. }( \pm) 1 \\
69 \text { cases }\end{array}$ & $1^{\prime} \mid \begin{array}{l}\text { A.R. }(-) 1^{\prime} \\
65 \text { cases }\end{array}$ & \\
\hline Average & 21.6 & 14.2 & 15.0 & 18.1 & 22.6 & 26.6 & 37.5 \\
\hline Maximum & 150.0 & 45.0 & 60.0 & 56.4 & 95.0 & 100.0 & 150.0 \\
\hline Minimum & 2.5 & 2.5 & 5.0 & 3.5 & 5.0 & 1.8 & 6.0 \\
\hline Average & 21.6 & & & & 22.4 & & 37.5 \\
\hline
\end{tabular}

2. The diastase content of the Arakawa-positive milk (Cf. Table II).

a. The diastase content of Arakawa's reaction of the intensity of (H) 1 1*.

55 cases were examined. It fluctuates from 2.5 to 45.0 ; the average is 14.2 .

b. The diastase content of Arakawa's reaction of the intensity of $(+1) 1^{\prime \dagger}$.

82 cases were examined. It fluctuatès from 5.0 to 60.0 ; the average is 15.0 .

c. The average of all the samples of the Arakawa-positive milk.

The average of diastase of Arakawa-positive milk is 14.8 .

* Arakawa's reaction of the intensity (H) in one minute.

+ Arakawa's reaction of the intensity $(H)$ in one minute. 
3. The diastase content of completely Arakawa-negative milk or milk with the intensity of reaction (-) $5^{\prime s}$ (Cf. Table II).

46 cases were examined. It fluctuates from 6.0 to 150.0 ; the average amounts to 37.9 .

4. The weakly Arakawa-positive.milk (clinically the Arakawa-negative milk) (Cf. Table II).

a. The diastase content of the milk of Arakawa's reaction of the intensity $;(+) 1^{\prime \prime}$.

63 cases were examined. It fluctuates from 3.5 to 56.4 ; the average is 18.1 .

b. The diastase content of the milk of Arakawa's reaction of the intensity, $( \pm) 1^{\prime * *}$.

69 cases were examined. It fluctuates from 5.0 to 95.0 ; the average is 22.6 .

c. The diastase content of the milk of Arakawa's reaction of the intensity $;(-) 1^{\prime} \ddagger$.

65 cases were examined. It fluctuates from 1.8 to 100.0 ; the average is 26.6 .

d. The average of all the milk samples under Item 4 or the average of the weakly Arakawa-positive milk is 22.4.

5. Distribution of all milk samples according to the diastase content and the intensity of A rakawa's reaction (Cf. Table III).

In Table III, all the milk samples are artanged according to the intensity of A rakawa's reaction. From Table III, we learn the following facts.

a. The diastase content of all the milk samples lies between 5.0 and 30.0 (79.0\% of all the cases) in the great majority of the cases. The largest number (86) of the cases lies between 10.0 and $15.0(22.6 \%)$.

b. In the group of A.R. (H) $1^{\prime}$ and A.R. (H)1', the diastase content is seen between 5.0 and 20.0 in the great majority of the cases $(79.4 \%)$. The most frequent figures were between 10.0 and 15.0 (38.9\%).

c. In the groups of A.R. $(+) 1^{\prime},( \pm) 1^{\prime}$, and $(-) 1^{\prime}, 56 \%$ of the cases has the diastase content between 10.0 and 25.0. The most frequent diastase values are seen between 15.0 and $20.0(23.8 \%)$.

d. In the group of completely Arakawa-positive milk, the most frequent figures of diastase content are seen between 35.0 and 40.0 , but the diastase values are distributed in a rather wide range in this group. If we simplify Table III (Cf. Table IV), dividing the diastase contents into two groups i.e. the group of $0-15.0$ and the group of 15.0-- ; we find in Table $V$ that the cases with the

5 Arakawa's reaction still negative after 5 minutes.

J Arakawa's reaction of the intensity $(t)$ in one minute.

** A rakawa's reaction of the intensity $( \pm)$ in one minute.

$\neq$ A rakawa's reaction of the intensity ( - ) in one minute. 
diastase contents over 15.0 in the group of A.R. (H) $1^{\prime}$ and A.R. (H)1' amount to $32 \%$, to $69.1 \%$ in the groups of A.R. $(+) 1^{\prime},( \pm) 1^{\prime}$ and A.R. $(-) 1^{\prime}$, and to $84.4 \%$ in the group of A.R. (-) $5^{\prime}$ respectively (Cf. Table IV). Based upon Table III, the curves are presented in Fig. 1, taking diastase content on axis of abscissa and numbers of $\%$ in each group of the milk of different A rakawa's reaction on the axis of ordinate.

TABLE III.

Distribution of all the milk samples according to the diastase content and the intensity of A rakawa's reaction.

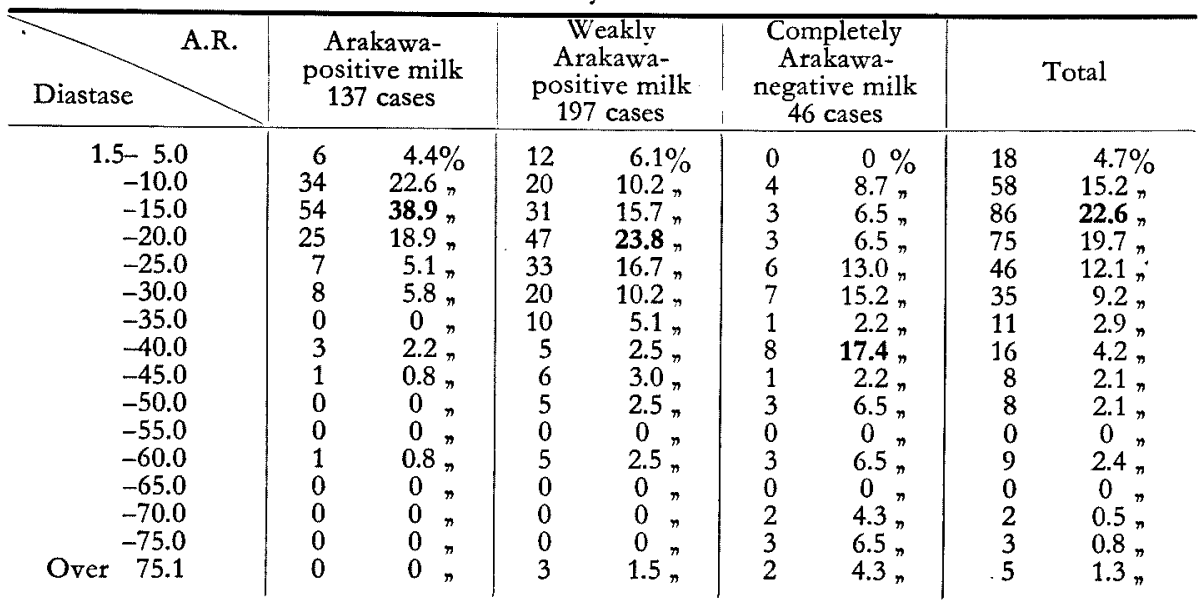

TABLE IV. (TABLE III, simplified.)

Distribution of all milk samples according to the intensity of A rakawa's reaction and diastase content.

\begin{tabular}{c|c|c|c|c}
\hline \multirow{2}{*}{ Diastase } & $\begin{array}{c}\text { Arakawa- } \\
\text { positive milk } \\
\text { A.R. (H) 1' } \\
\text { A.R. (H) 1' }\end{array}$ & $\begin{array}{c}\text { Weakly Arakawa- } \\
\text { positive milk } \\
\text { A.R. }(+) 1^{\prime} \\
\text { A.R. }( \pm) 1^{\prime} \\
\text { A.R. (-) } 1^{\prime}\end{array}$ & $\begin{array}{c}\text { Completely } \\
\text { Arakawa- } \\
\text { negative milk } \\
\text { A.R. (-) 5' }\end{array}$ & Total \\
\hline Under 15.0 & $\begin{array}{c}92 \text { cases } \\
(67.1 \%)\end{array}$ & $\begin{array}{c}63 \text { cases } \\
(31.9 \%)\end{array}$ & $\begin{array}{c}7 \text { cases } \\
(15.2 \%)\end{array}$ & $\begin{array}{c}162 \text { cases } \\
(42.6 \%)\end{array}$ \\
Over 15.1 & $\begin{array}{c}45 \text { cases } \\
(32.9 \%)\end{array}$ & $\begin{array}{c}134 \text { cases } \\
(69.1 \%)\end{array}$ & 39 cases & 218 cases \\
Tota! & 137 cases & 197 cases & 46 cases & 380 cases
\end{tabular}

6. Cases of one and the same lactants with different Arakawa's reaction on different sides of the breast (CF. Table V).

Of all the 136 mothers, 85 cases $(62.3 \%)$ have a larger diastase content on the side of the weaker A rakawa's reaction than on the side of the stronger. In 23 cases $(16.9 \%$ ) of all the mothers, the result was quite the reverse. 28 cases $(20.8 \%)$ have an about equal diastase content in spite of different Arak awa's reaction. 
Fig. 1. Curves showing the distribution of milk samples according to the intensity of A ra kawa's reaction and diastase content.

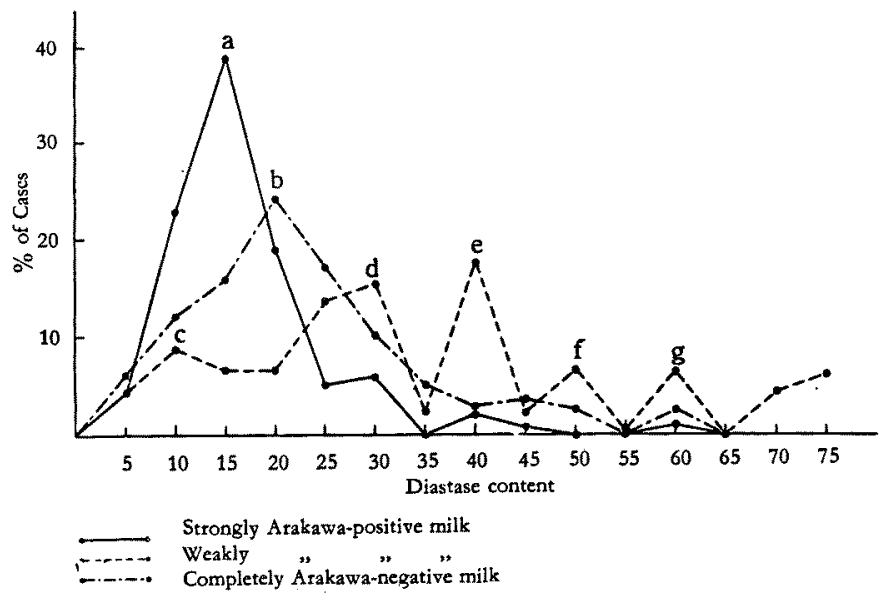

TABLE V.

Diastase and different A rakawa's reaction of the milk which is secreted from different sides of the breasts.

\begin{tabular}{c|c|c}
\hline $\begin{array}{c}\text { Number of cases with the larger } \\
\text { diastase content on the side of } \\
\text { the weaker A r a ka wa's } \\
\text { reaction }\end{array}$ & $\begin{array}{c}\text { Number of cases with the larger } \\
\text { diastase content on the side of } \\
\text { the weaker A rak a wa's } \\
\text { reaction }\end{array}$ & $\begin{array}{c}\text { Number of cases with different } \\
\text { A ra k a w a's reaction with } \\
\text { equal diastase content }\end{array}$ \\
\hline \begin{tabular}{c|c}
85 cases \\
$(62.4 \%)$
\end{tabular} & $\begin{array}{c}23 \text { cases } \\
(16.8 \%)\end{array}$ & 28 cases \\
\hline
\end{tabular}

7. Cases of one and the same lactants with equal Arakawa's reaction on different side of the breast (Cf. Table VI).

Of all the 53 cases, 34 cases $(64.0 \%)$ have different diastase content, but the difference, as a rule, remains small though there are of course a few exceptions.

TABle VI.

Diastase in the case of the same A rakawa's reaction in different sides of the breasts.

\begin{tabular}{c|c}
\hline $\begin{array}{c}\text { Number of cases with equal A rakawa's } \\
\text { reaction on both side, but with different } \\
\text { diastase content on different sides }\end{array}$ & $\begin{array}{c}\text { Number of cases with equal Araka wa's } \\
\text { reaction and with equal diastase } \\
\text { content on different sides }\end{array}$ \\
\hline 34 cases & 19 cases \\
$(64 \%)$ & $(36 \%)$
\end{tabular}

8. Diastase content of the milk secreted from healthy mothers with healthy sucklings (Cf. Tables VII and VIII). 
Milk samples were collected from the mothers who brought their babies to "Baby show" which was held on May 5th, 1934. Method of experiment was as usual, except that the diastase had been estimated mixing equal quantities of milk from each cases instead of to determine respectively. The diastase value was 14.1 for the group of A.R. (\#) $1^{\prime}$ and $(+) 1^{\prime}$, and was 17.6 for the group of A.R. $(+) 1^{\prime},( \pm) 1^{\prime}$ and $(-) 1^{\prime}$. In the case of mixing the milk of all the different reaction, diastase value was 15.0. Comparison of the milk

TABle VII.

Diastase content of milk samples obtained in the "Baby show".

\begin{tabular}{c|c|c|c}
\hline $\begin{array}{c}\text { Arakawa's } \\
\text { reaction }\end{array}$ & $\begin{array}{c}\text { All the milk samples } \\
\text { including different } \\
\text { reaction } \\
113 \text { cases }\end{array}$ & $\begin{array}{c}\text { Arakawa-positive } \\
\text { milk } \\
63 \text { cases }\end{array}$ & $\begin{array}{c}\text { Arakawa-negative } \\
\text { milk } \\
24 \text { cases }\end{array}$ \\
\hline Diastase & 15.0 & 14.1 & 17.1
\end{tabular}

TABLE VIII.

Comparison of the milk diastase content in our Pediatric Out-Patient Department and in the "Baby show".

\begin{tabular}{c|c|c|c}
\hline $\begin{array}{c}\text { Arakawa's } \\
\text { reaction } \\
\text { Milk }\end{array}$ & All milk samples & positive milk & negative milk \\
\cline { 2 - 4 } $\begin{array}{c}\text { Our Pediatric } \\
\text { Dispensary }\end{array}$ & 21.6 & 14.8 & 25.5 \\
\hline The "Baby show" & 15.0 & 14.1 & 17.0
\end{tabular}

diastase figures obtained in our Pediatric Dispensary and those obtained in the "Baby show" is listed in Table VIII. 14.8 and 14.1 mean almost the same amount of diastase, while 25.5 and 17.0 mean very different amounts of it.

9. Diastase in colostrum (Cf. Table IX).

Diastase is very abundant in colostrum. The maximum 882.0 and the minimum 42.9. The average is 256.8 . As to the relation toward A rakawa's reaction, diastase is thus rich in the milk of weaker Arakawa's reaction than in that of the stronger reaction, though the cases are a few in number.

TABle IX.

Diastase in colostrum (within 7 days after the delivery).

\begin{tabular}{|c|c|c|}
\hline No. & $\begin{array}{c}\text { Arakawa's reaction } \\
0^{\prime} 1^{\prime} 2^{\prime} 3^{\prime} 4^{\prime} 5^{\prime}\end{array}$ & Diastase \\
\hline 1 & $\begin{array}{l}\mathrm{R}+\mathrm{H} H \mathrm{H}+\mathrm{H}+\mathrm{H} \\
\mathrm{L}+\mathrm{H} H+H \mathrm{H} H\end{array}$ & $\begin{array}{l}46.1 \\
46.1\end{array}$ \\
\hline 2 & $\begin{array}{l}\mathrm{R}--\overline{-}-\overline{-} \\
\mathrm{L}-----\end{array}$ & $\begin{array}{l}166.4 \\
138.8\end{array}$ \\
\hline 3 & $\begin{array}{l}R--+H+H \\
L--+H+H\end{array}$ & $\begin{array}{l}112.8 \\
103.6\end{array}$ \\
\hline 4 & $\begin{array}{l}\mathrm{R}=-\overline{+}+++ \\
\mathrm{L}-+\mathrm{H} H+\mathrm{H}\end{array}$ & $\begin{array}{c}42.9 \\
*\end{array}$ \\
\hline 5 & $\begin{array}{l}\mathrm{R}----- \pm \\
\mathrm{L}---- \pm \pm\end{array}$ & $\begin{array}{l}882.0 \\
859.0\end{array}$ \\
\hline 6 & $\begin{array}{l}\mathrm{R}-ー- \pm \pm \pm \\
\mathrm{L}---ー \pm \pm\end{array}$ & $\begin{array}{l}329.0 \\
333.0\end{array}$ \\
\hline 7 & $\begin{array}{l}\mathrm{R}+\mathrm{H}+\mathrm{H}+\mathrm{H}+\mathrm{H} \\
\mathrm{L}- \pm++\mathrm{H} \\
\text { erage of Diastase } 256 \\
\text { estimated. }\end{array}$ & $\begin{array}{l}375.0 \\
500.0\end{array}$ \\
\hline
\end{tabular}


10. Diastase and age of sucklings (Cf. Table $\mathrm{X}$ ).

Table $\mathrm{X}$ shows the relation between milk diastase and age of sucklings. In early months of an infant, diastase seems to have higher value and then gradually decreases its value, but again, when the age of an infant becomes larger such as 9-10 months or more, milk diastase seems to increases. These inclinations can be also seen in the group of milk positive to A rakawa's reaction and in that of milk negative to it (Cf. Table X).

TABLE $X$.

Diastase and age of sucklings.

\begin{tabular}{c|c|c|c|c|c|c}
\hline \multirow{2}{*}{$\begin{array}{c}\text { Age of } \\
\text { scukling } \\
\text { m=month }\end{array}$} & \multicolumn{2}{|c|}{$\begin{array}{c}\text { All the cases } \\
\text { examined }\end{array}$} & \multicolumn{2}{c|}{$\begin{array}{c}\text { Arakawa-positive } \\
\text { milk }\end{array}$} & \multicolumn{2}{c}{$\begin{array}{c}\text { Arakawa-negative } \\
\text { milk }\end{array}$} \\
\cline { 2 - 6 } \cline { 5 - 6 } & Diastase & $\begin{array}{c}\text { Numbers of } \\
\text { examples }\end{array}$ & Diastase & $\begin{array}{c}\text { Numbers of } \\
\text { examples }\end{array}$ & Diastase & $\begin{array}{c}\text { Numbers of } \\
\text { examples }\end{array}$ \\
\hline $0 \mathrm{~m}-2 \mathrm{~m}$ & 35.8 & 66 & 21.6 & 5 & 36.9 & 61 \\
$-4 \mathrm{~m}$ & 21.0 & 64 & 14.6 & 22 & 24.9 & 42 \\
$-6 \mathrm{~m}$ & 15.9 & 55 & 11.9 & 21 & 18.6 & 34 \\
$-8 \mathrm{~m}$ & 18.6 & 43 & 12.1 & 20 & 24.2 & 23 \\
$-10 \mathrm{~m}$ & 16.4 & 47 & 14.3 & 23 & 18.1 & 24 \\
$-12 \mathrm{~m}$ & 19.4 & 27 & 15.4 & 17 & 26.3 & 10 \\
Over 1 year & 20.9 & 77 & 18.8 & 27 & 21.9 & 50
\end{tabular}

11. Diastase and age of mothers (Cf. Table XI).

From Table XI, we see that younger mothers secrete more diastase into milk than the older ones. This is contrary to my expectation, because I have expected that milk of older mothers may be larger in diastase content than that of younger ones, if both are of the same group of A rakaw a's reaction.

TABLE XI.

Diastase content according to the age of mothers.

\begin{tabular}{c|c|c|c}
\hline $\begin{array}{c}\text { Years } \\
\text { of age }\end{array}$ Diastase & $\begin{array}{c}\text { All the milk } \\
\text { samples }\end{array}$ & $\begin{array}{c}\text { Arakawa-position } \\
\text { milk }\end{array}$ & $\begin{array}{c}\text { Arakawa-negative } \\
\text { milk }\end{array}$ \\
\hline Under 25 & 25.2 & 15.5 & 27.2 \\
\hline from 26 to 30 & 21.4 & 14.8 & 25.8 \\
\hline Over 31 & 19.8 & 11.5 & 24.6
\end{tabular}

12. Variation of diastase content before and after feeding (Cf. Table XII).

Milk portion after feeding is richer in diastase content than that before it.

13. Mastitis and milk diastase (Cf. Table XIII).

Milk secreted from a gland affected with mastitis or has an anamnesis of mastitis is richer in diastase content even though the local disease of a considerably old date. 
Table XII.

Diastase content before and after feeding.

\begin{tabular}{|c|c|c|c|c|c|}
\hline No. & Name & $\begin{array}{l}\text { Arakawa's } \\
\text { reaction } \\
\text { before feeding } \\
0^{\prime} 1^{\prime} 2^{\prime} 3^{\prime} 4^{\prime} 5^{\prime}\end{array}$ & $\begin{array}{l}\text { Arakawa's } \\
\text { reaction } \\
\text { after feeding } \\
0^{\prime} 1^{\prime} 2^{\prime} 3^{\prime} \quad 4^{\prime} \quad 5^{\prime}\end{array}$ & $\begin{array}{l}\text { Diastase } \\
\text { before } \\
\text { feeding }\end{array}$ & $\begin{array}{l}\text { Diastase } \\
\text { after } \\
\text { feeding }\end{array}$ \\
\hline 1 & Sato & 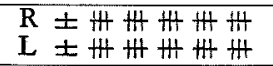 & $\begin{array}{l}R \pm \# \text { \#\# \# \# } \\
\mathrm{L} \pm \text { \# H H H }\end{array}$ & $\begin{array}{l}13.0 \\
14.0\end{array}$ & $\begin{array}{l}13.3 \\
16.6\end{array}$ \\
\hline 2 & Onodera & $\begin{array}{l}R+H+H H H \\
\mathbf{L} \pm H+H W H\end{array}$ & 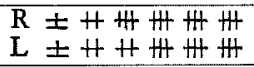 & $\begin{array}{l}15.0 \\
13.6 \\
\end{array}$ & $\begin{array}{l}15.0 \\
14.0\end{array}$ \\
\hline 3 & Otomo & $\begin{array}{l}\cdot \mathbf{R} \pm \# \text { H H H H } \\
\cdot \mathbf{L}=\mathrm{H} H \mathrm{H} H \mathrm{H}\end{array}$ & $\begin{array}{l}\mathrm{R} \pm \#+\# \text { H H } \\
\mathrm{L}-++\mathrm{H} \text { H }\end{array}$ & $\begin{array}{l}15.0 \\
15.0\end{array}$ & $\begin{array}{l}16.6 \\
23.0\end{array}$ \\
\hline 4 & Sindo & $\begin{array}{l}\mathrm{R}-+H+H+H \\
\mathrm{~L}- \pm-- \pm\end{array}$ & $\begin{array}{l}\mathrm{R}-ー- \pm \pm \pm \\
\mathrm{L}--- \pm\end{array}$ & $\begin{array}{l}4.1 \\
6.8 \\
\end{array}$ & $\begin{array}{l}6.0 \\
7.9 \\
\end{array}$ \\
\hline 5 & Okuro & $\begin{array}{l}R--\overline{-}-\overline{-} \\
\mathrm{L}- \pm \pm \pm \pm \pm\end{array}$ & 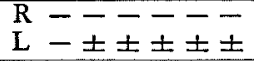 & $\begin{array}{l}37.5 \\
27.3\end{array}$ & $\begin{array}{l}46.1 \\
27.1\end{array}$ \\
\hline 6 & Ito & $\begin{array}{l}\mathrm{R}-\overline{-}-\overline{-} \\
\mathrm{L}- \pm \pm \pm \pm\end{array}$ & $\begin{array}{l}\mathrm{R}-\overline{-}-\overline{-} \\
\mathrm{L}-\overline{ \pm}\end{array}$ & $\begin{array}{l}5.0 \\
5.0 \\
\end{array}$ & $\begin{array}{l}7.1 \\
5.8\end{array}$ \\
\hline 7 & Aoki & $\begin{array}{l}\mathbf{R}--\overline{-}- \\
\mathbf{L}-----\end{array}$ & $\begin{array}{l}\mathrm{R}------ \\
\mathrm{L}-----\end{array}$ & $\begin{array}{r}100.0 \\
60.0\end{array}$ & $\begin{array}{r}100.0 \\
64.0\end{array}$ \\
\hline 8 & Abe & $\begin{array}{l}\mathrm{R}-- \pm-\overline{-} \\
\mathrm{L}-- \pm \pm \pm\end{array}$ & $\begin{array}{l}\mathrm{R}-\overline{-}-\overline{-} \\
\mathrm{L}-\overline{ \pm}\end{array}$ & $\begin{array}{l}30.0 \\
27.3 \\
\end{array}$ & $\begin{array}{l}28.5 \\
29.5 \\
\end{array}$ \\
\hline 9 & Tiba & $\begin{array}{l}\mathrm{R}----\overline{-} \\
\mathrm{L}-----\end{array}$ & $\begin{array}{l}\mathrm{R}---\overline{-} \\
\mathrm{L}-----\end{array}$ & $\begin{array}{l}36.0 \\
36.0\end{array}$ & $\begin{array}{l}37.5 \\
37.5\end{array}$ \\
\hline 10 & Senda & $\begin{array}{l}\mathrm{R}- \pm+H+H \\
\mathrm{~L}-+H \text { H H H }\end{array}$ & $\begin{array}{l}R- \pm+H+H \\
L \pm+H \text { H H }\end{array}$ & $\begin{array}{l}23.7 \\
16.6\end{array}$ & $\begin{array}{l}27.3 \\
16.6\end{array}$ \\
\hline 11 & Taka & $\begin{array}{l}\mathrm{R}-\overline{-} \overline{\mathrm{I}} \overline{+} \\
\mathrm{L}-\overline{+}\end{array}$ & $\begin{array}{l}\mathrm{R}-ー-ニ-\bar{~} \\
\mathrm{~L}-ー \pm \pm \pm \pm\end{array}$ & $\begin{array}{r}150.0 \\
25.0\end{array}$ & $\begin{array}{r}164.6 \\
31.6\end{array}$ \\
\hline 12 & Yosida & $\begin{array}{l}\mathrm{R}--++++ \\
\mathrm{L}- \pm++++\end{array}$ & $\begin{array}{l}\mathrm{R}-- \pm \pm++ \\
\mathrm{L}- \pm+\mathrm{H}\end{array}$ & $\begin{array}{l}25.0 \\
23.1\end{array}$ & $\begin{array}{l}27.0 \\
23.1\end{array}$ \\
\hline 13 & Sasaki & $\begin{array}{l}\mathrm{R}-+H+H \\
\mathrm{~L} \pm H+H\end{array}$ & $\begin{array}{l}\mathrm{R}-\overline{-}+++ \\
\mathrm{L}-+\mathrm{H}+\mathrm{H}\end{array}$ & $\begin{array}{l}21.5 \\
16.7 \\
\end{array}$ & $\begin{array}{l}27.3 \\
19.6 \\
\end{array}$ \\
\hline 14 & Mayama & $\begin{array}{l}\mathrm{R}-++H+\# \\
\mathrm{~L}- \pm+H+H\end{array}$ & $\begin{array}{l}\mathrm{R}-\overline{-} \overline{-} \overline{\mathrm{H}} \\
\mathrm{L}-\bar{H}\end{array}$ & $\begin{array}{l}15.0 \\
16.0\end{array}$ & $\begin{array}{l}30.0 \\
20.0\end{array}$ \\
\hline
\end{tabular}

\section{TABLE XIII.}

Diastase content of the milk secreted from breasts which have anamnesis of mastitis.

\begin{tabular}{|c|c|c|c|c|}
\hline No. & Name & 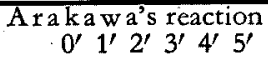 & Diastase & Remarks \\
\hline 1. & C. Kumagai & $\begin{array}{l}\mathrm{R}-H \text { H H H H } \\
\mathrm{L} \pm \text { H H H H }\end{array}$ & $\begin{array}{r}60.0 \\
2.5 \\
\end{array}$ & $\begin{array}{l}\text { Mastitis on right side before } \\
3 \text { years }\end{array}$ \\
\hline 2 & A. Sato & $\begin{array}{l}R=-\overline{-}-\bar{H} \\
\mathrm{~L}- \pm+H\end{array}$ & $\begin{array}{l}66.7 \\
28.1\end{array}$ & $\begin{array}{l}\text { Mastitis on right side before } \\
4 \text { years }\end{array}$ \\
\hline 3 & F. Kaneko & $\begin{array}{l}R=H+H \text { H H } \\
L \pm H H H+H\end{array}$ & $\begin{array}{l}11.5 \\
12.2\end{array}$ & $\begin{array}{l}\text { Mastitis on left side before } \\
12 \text { years }\end{array}$ \\
\hline 4 & T. Tanno & $\begin{array}{l}\mathrm{R}--\overline{-}-\overline{-} \\
\mathrm{L}-----\end{array}$ & $\begin{array}{l}40.0 \\
37.5\end{array}$ & Mastitis on both side now \\
\hline 5 & Y. Sindo & $\begin{array}{l}\mathrm{R}=+\mathrm{H} H \mathrm{H} \\
\mathrm{L}=--- \pm\end{array}$ & $\begin{array}{r}8.3 \\
13.6\end{array}$ & $\begin{array}{l}\text { Mastitis on left side before } \\
3 \text { years }\end{array}$ \\
\hline 6 & E. Otuka & $\begin{array}{l}\mathrm{R} \pm \#+\#+\# \\
\mathrm{~L}= \pm \pm \pm \pm+\end{array}$ & $\begin{array}{l}11.1 \\
32.2 \\
\end{array}$ & $\begin{array}{l}\text { Mastitis on left side before } \\
5 \text { years }\end{array}$ \\
\hline 7 & T. Sato & $\begin{array}{l}R= \pm+\#+H \\
L \pm \#+\# H+H\end{array}$ & $\begin{array}{l}21.5 \\
12.0\end{array}$ & $\begin{array}{l}\text { Mastitis on right side before } \\
10 \text { years }\end{array}$ \\
\hline
\end{tabular}

Mean value of the diastase of mastitis milk 
14. Diastase and fat content of milk (Cf. Tables XIV, XV and XVI).

As my cases are not numerous, it may be too hasty to draw a difinite conclusion, but it seems to me that milk richer in diastase content contains a larger. amount of fat as far as my experience goes.

TABLE XIV.

Diastase, fat and Arakawa's reaction of milk.

\begin{tabular}{|c|c|c|c|c|c|c|c|}
\hline $\begin{array}{l}\text { No. (Cf. } \\
\text { Table 1) }\end{array}$ & A.R. & Diastase & Fat & No. & A.R. & Diastase & Fat \\
\hline $\begin{array}{l}181 \\
182 \\
\end{array}$ & $\begin{array}{l}16(-) \\
14( \pm)\end{array}$ & $\begin{array}{l}37.5 \\
27.5 \\
\end{array}$ & $\begin{array}{l}4.9 \% \\
2.5 \% \\
\end{array}$ & $\begin{array}{l}213 \\
214 \\
\end{array}$ & $\begin{array}{c} \pm \pm+H+H \\
1(H)\end{array}$ & $\begin{array}{r}21.5 \\
8.1 \\
\end{array}$ & $\begin{array}{l}1.2 \% \\
3.2 \%\end{array}$ \\
\hline $\begin{array}{l}185 . \\
186 \\
\end{array}$ & $\begin{array}{r}8(-) \\
11(+) \\
\end{array}$ & $\begin{array}{l}21.5 \\
18.8 \\
\end{array}$ & $\begin{array}{l}3.1 \% \\
2.4 \pi\end{array}$ & $\begin{array}{l}215 \\
216 \\
\end{array}$ & $\begin{array}{l}1(\#) \\
1(\#)\end{array}$ & $\begin{array}{l}13.6 \\
16.6\end{array}$ & $\begin{array}{l}2.7 \% \\
4.6 \%\end{array}$ \\
\hline $\begin{array}{l}187 \\
188\end{array}$ & $\begin{array}{l}1(H) \\
1(H)\end{array}$ & $\begin{array}{l}8.8 \\
8.8\end{array}$ & $\begin{array}{l}1.3 \pi \\
3.1 n\end{array}$ & $\begin{array}{c}* \\
218\end{array}$ & * & $\begin{array}{c}* \\
14.2\end{array}$ & 4.1 \\
\hline $\begin{array}{l}191 \\
192\end{array}$ & $\begin{array}{r}6(-) \\
12(-)\end{array}$ & $\begin{array}{l}16.6 \\
18.8\end{array}$ & $\begin{array}{l}3.2 n \\
4.1 n\end{array}$ & $\begin{array}{l}219 \\
220\end{array}$ & $\begin{array}{l}8(-) \\
6( \pm)\end{array}$ & $\begin{array}{l}16.6 \\
16.6\end{array}$ & $\begin{array}{l}2.7 n \\
1.4 n\end{array}$ \\
\hline $\begin{array}{l}195 \\
196\end{array}$ & $\begin{array}{r}12(-) \\
4(+)\end{array}$ & $\begin{array}{l}60.0 \\
20.0\end{array}$ & $\begin{array}{l}5.3 n \\
4.1 n\end{array}$ & $\begin{array}{l}227 \\
228\end{array}$ & $-\frac{-7}{16(-)}$ & $\begin{array}{l}25.0 \\
16.6\end{array}$ & $\begin{array}{l}3.7 " \\
1.2 \%\end{array}$ \\
\hline $\begin{array}{l}197 \\
198\end{array}$ & $\begin{array}{l}12(-) \\
15(-)\end{array}$ & $\begin{array}{l}25.0 \\
27.0\end{array}$ & $3.9 n$ & $\begin{array}{l}255 \\
256\end{array}$ & $\begin{array}{l}6( \pm) \\
7( \pm)\end{array}$ & $\begin{array}{l}46.1 \\
46.1\end{array}$ & $\begin{array}{l}3.6 \pi \\
3.6 n\end{array}$ \\
\hline $\begin{array}{l}201 \\
202\end{array}$ & $\begin{array}{l}1(H) \\
1(H)\end{array}$ & $\begin{array}{l}15.0 \\
15.0\end{array}$ & $\begin{array}{l}3.3 \text { " } \\
3.2 \text { " }\end{array}$ & $\begin{array}{l}277 \\
278\end{array}$ & $\begin{array}{l}1(H) \\
1(H)\end{array}$ & $\begin{array}{l}10.0 \\
10.0\end{array}$ & $3.1 "$ \\
\hline $\begin{array}{l}203 \\
204 \\
\end{array}$ & $\begin{array}{l}2( \pm) \\
1(H) \\
\end{array}$ & $\begin{array}{l}21.5 \\
12.0 \\
\end{array}$ & $\begin{array}{l}6.4 \% \\
1.4 \% \\
\end{array}$ & $\begin{array}{l}281 \\
282 \\
\end{array}$ & $\begin{array}{r}16(-) \\
6(-)\end{array}$ & $\begin{array}{l}15.0 \\
25.0\end{array}$ & $\begin{array}{l}4.8 \% \\
3.7 \%\end{array}$ \\
\hline $\begin{array}{l}207 \\
208 \\
\end{array}$ & $\begin{array}{c} \pm+H \# H \\
1(H)\end{array}$ & $\begin{array}{l}5.0 \\
5.0 \\
\end{array}$ & $\begin{array}{l}3.8 \pi \\
0.4 \%\end{array}$ & $\begin{array}{l}283 \\
284\end{array}$ & $\begin{array}{c}++t+ \\
8(-)\end{array}$ & $\begin{array}{l}25.0 \\
23.1\end{array}$ & $\begin{array}{l}2.4 n \\
1.3_{n}^{n}\end{array}$ \\
\hline $\begin{array}{l}209 \\
210 \\
\end{array}$ & $\begin{array}{l}1(\text { H) } \\
1(\text { H) } \\
\end{array}$ & $\begin{array}{r}13.6 \\
7.5 \\
\end{array}$ & $\begin{array}{l}1.4 n \\
2.3^{n} \\
\end{array}$ & $\begin{array}{c}* \\
286\end{array}$ & $12(-)$ & $\begin{array}{c}* \\
20.0\end{array}$ & $\begin{array}{r}* \\
2.8\end{array}$ \\
\hline $\begin{array}{l}211 \\
212\end{array}$ & $\begin{array}{r}15(-) \\
8(-)\end{array}$ & $\begin{array}{l}5.0 \\
5.0\end{array}$ & $\begin{array}{l}0.4 \text { " } \\
0.7 \text { n }\end{array}$ & & & & \\
\hline
\end{tabular}

TABle XV.

Fat content according to the diastase content.

\begin{tabular}{c|l}
\hline $\begin{array}{c}\text { Diastase } \\
\text { ovet 15.1 }\end{array}$ & $\left.\begin{array}{l}\text { Mean fat } \\
\text { content }\end{array}\right\} 3.36 \%$ \\
\hline $\begin{array}{c}\text { Diastase } \\
\text { under 15.0 }\end{array}$ & $\left.\begin{array}{l}\text { Mean fat } \\
\text { content }\end{array}\right\} 2.31 \%$
\end{tabular}

TABle XVI.

Fat content according to the intensity of A rakawa's reaction.

\begin{tabular}{c|c}
\hline $\begin{array}{c}\text { Mean fat content } \\
\text { of Arakawa- } \\
\text { positive milk }\end{array}$ & $2.69 \%$ \\
\hline $\begin{array}{c}\text { Mean fat content } \\
\text { of Arakawa- } \\
\text { negative milk }\end{array}$ & $3.16 \%$ \\
\hline
\end{tabular}

15. Diastase in the case of menstruation, pregnancy and syphilis (Cf. Tables XVII and XVIII).

From Tables XVII and XVIII, diastase in the milk secreted from mothers during menstrual period is slightly larger than the mean value of the enzyme in all the milk samples of lactating women. Milk diastase seems also to show a higher value in the case of syphilitic women. The cases are however only too few in number to state something definite.

But the following experience of mine obtained in the city hospital in 
TABle XVII.

Diastase of milk and syphilitis.

\begin{tabular}{|c|c|c|c|c|}
\hline No. & Name & $\begin{array}{c}\text { Arakawas reaction } \\
0^{\prime} 1^{\prime} 2^{\prime} 3^{\prime} 4^{\prime} 5^{\prime}\end{array}$ & Diastase & Remarks \\
\hline $\begin{array}{l}39 \\
40\end{array}$ & N. T. & 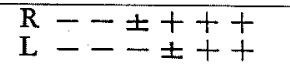 & $\begin{array}{r}42.8 \\
.37 .5\end{array}$ & \\
\hline $\begin{array}{l}119 \\
120\end{array}$ & Y. K. & $\begin{array}{l}\mathrm{R}=-ー-ー- \\
\mathrm{L}-ー-ー\end{array}$ & $\begin{array}{l}75.0 \\
75.0\end{array}$ & \\
\hline $\begin{array}{l}183 \\
184\end{array}$ & W. K. & $\begin{array}{l}\mathrm{R}=--ー- \pm \\
\mathrm{L}-ー-ー \pm \pm\end{array}$ & $\begin{array}{l}33.4 \\
27.6\end{array}$ & $\begin{array}{l}\text { Wassermann ( } \#) \\
\text { Blood platelet } 61 \text { millions. } \\
\text { Methyl glyoxal } \begin{cases}R & 1.1 \text { mgrms. } \\
L & 1.76 \text { mgrms. }\end{cases} \\
\text { Fat } \begin{cases}R & 4.85 \% \\
L & 2.50 \%\end{cases} \end{array}$ \\
\hline $\begin{array}{l}276 \\
268\end{array}$ & H. C. & $\begin{array}{l}\mathrm{R}--ー-ー- \\
\mathrm{L}----\div\end{array}$ & $\begin{array}{l}36.1 \\
36.1\end{array}$ & \\
\hline $\begin{array}{l}355 \\
356\end{array}$ & K. M. & $\begin{array}{r}R=- \pm \pm++ \\
\mathbf{R}- \pm+H+H \\
\text { Average }\end{array}$ & $\begin{array}{l}21.5 \\
18.8 \\
40.4\end{array}$ & 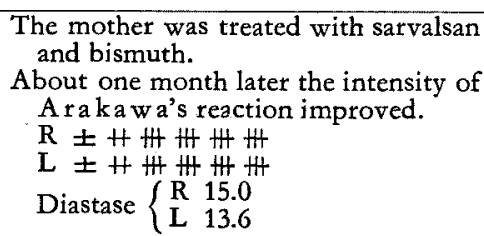 \\
\hline
\end{tabular}

Muroran, Hokkaido, may be an instructive one.

Case report. Infant $\circ 7$

TABle XVIII.

Diastase and menstruation. months, Os aw a by name.

On June, 25th, 1936, a mother visited oúr Pediatric Dispensary with the complainment of dyspeptic stools of her child. It was cured without any specific treatment within a few days. Arakawa's reaction of her milk at that time was on the right side $1(H) 1^{\prime *}$

\begin{tabular}{|c|c|c|c|}
\hline No. & Name & 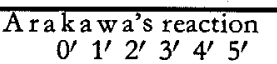 & Diastase \\
\hline $\begin{array}{l}133 \\
134\end{array}$ & T. H. & 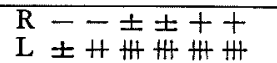 & $\begin{array}{l}28.5 \\
13.6\end{array}$ \\
\hline $\begin{array}{l}111 \\
112\end{array}$ & M. T. & $\begin{array}{l}\mathrm{R}=-ニ-ニ \pm \\
\mathrm{L}=-ニ- \pm\end{array}$ & $\begin{array}{l}25.0 \\
27.6\end{array}$ \\
\hline $\begin{array}{l}159 \\
\cdot 160\end{array}$ & Z.W. & $\begin{array}{l}\mathrm{R}=-ー \pm++ \\
\mathrm{L}=- \pm+++\end{array}$ & $\begin{array}{l}50.0 \\
42.8\end{array}$ \\
\hline $\begin{array}{l}199 \\
200\end{array}$ & E. N. & $\begin{array}{l}R \pm H+H \# \text { H } \\
L \pm H \# H \# H\end{array}$ & $\begin{array}{r}15.0 \\
8.8\end{array}$ \\
\hline $\begin{array}{l}291 \\
292\end{array}$ & X. $\mathrm{O}$ & $\begin{array}{l}\mathrm{R}-ー-ー- \pm \\
\mathrm{L}---ー \pm \pm\end{array}$ & $\begin{array}{l}12: 0 \\
12.0\end{array}$ \\
\hline & & Average & 23.5 \\
\hline
\end{tabular}
and on the left side $1(H) 1$ '. On. July 20 th, she came to me because her infant had again dyspeptic stools and asked: "Is the milk during menses not harmful to an infant?" Actually, this intelligent mother was in her period at that time. Then I became convinced that the cause of her infant's dyspepsia in June was highly probably due to the chemical change of milk caused by menses. Much interested in this problem, I examined A rak a wa's reaction and diastase content of her milk. A raka wa's reaction: right $6(+)$ and left $5( \pm)$. Diastase: right 18.8 and left 21.5. On August 7th, when her menstruation was over, A rakawa's reaction was on the right side $1(+)$ and on the left $3(+)$ and

* It is not difficult to presume that her A ra k a wa's reaction must have been much weaker in het premenstrual or menstrual period. 
diastase content was on the right 16.6 and on the left 18.8. On September 10th, she came to me complaining of her infant's bad stools again, and told me that the expected menses in August did not occur. Then I sent her to the Obsterical Department because I suspected that she might be in a new pregnancy, but the clinical evidence was not furnished by that department, because of a too early stage. At that time, Arakawa's reaction was: right $12(-)$ and left $14(-)$. Diastase was on the right 30.0 and on the left 30.0. I wanted to ascertain the existence of a new pregnancy by way of biological method and I injected 20 c.c. of her urine into the auricular vein of a virgin rabbit. 24 hours later, I examined the ovaries in which bleeding spots were observed distinctly. Her new pregnancy was thus certified. In this case diastase content of milk was rich during menstruation and pregnancy as was expected.

16. Influence of vitamin $B_{1}$ on milk diastase in vitro.

Adding 0.5 mgrms. of vitamin $B_{1}$ to milk in the buffer solution, the chemical examination was performed as usual, but no significant influence was observed in any case* on the diastase of human milk as far as my experiment goes.

17. Diastase and state of secretion of milk (Cf. Table XIX).

- It seems to show that the breast, which is poorer in secretion, secretes diastase richer milk and vice versa.

TABLE XIX.

Diastase content according to the state of secretion.

\begin{tabular}{c|c|c}
\hline \multicolumn{2}{c|}{$\begin{array}{c}\text { Cases of different state of secretion in same } \\
\text { body (49 cases) }\end{array}$} & $\begin{array}{c}\text { Hypogalactia of both } \\
\text { sides (35 cases) }\end{array}$ \\
\hline Bad side of secretion & Good side of secretion & Mean diastase value \\
Mean diastase value & Mean diastase value \\
28.8 & 20.0 & 23.9 \\
\hline
\end{tabular}

\section{Discussion.}

With respect to the origin and the raison d'être of diastase in human milk, there are many opinions. As is well known, starch, the object of diastatic action, is not identified in human milk and a breast fed infant does not take starch until or unless artificially fed. Some authors believe that milk diastase is of use for the nutrition of an breast fed infant, but the others are opposed to the opinion. For example, studying the diastase content of the tissue of mammary gland itself, Grimmer ${ }^{\mathbf{1 0}}$ ) saw richer diastase content in the not

* This experiment was done in 1940. I tried it with about 10 milk samples of different Arakawa's reaction.

10) W. Grimmer, Biochem. Ztschr., 1913, 53, 429. 
lactating gland. He concluded that milk diastase is an original enzyme of the mammary gland but he did not ascribe any nutritional significance to it, because milk did not contain starch. He assumed the diastase as a product of mammary gland which was necessary for milk production, and he supposed a certain part played by diastase in the milk sugar production. $\mathrm{Fu} \mathrm{jii}^{\mathbf{6}}$ ) is ${ }^{-}$of opinion that the milk diastase is originated haematogenously and is prepared for the partake of different nourishment and that it has a very important significance for infant in the period of weaning. Yoshiya et al ${ }^{11}$ also of almost the same opinion. But the abundant content of diastase in the colostrum will not be explained by this theory for a breast fed newborn. As $\mathrm{Fu} \mathrm{uii}^{6}$ ) and others state, milk diastase keeps a higher value as compared with blood diastase (serum diastase : milk diastase $=1: 20$ according to $\left.\mathrm{Fu} \mathrm{jii}{ }^{6}\right)$ ). So I believe that the milk diastase is not only produced by filtration from blood but also produced by active secretion of the mammary gland itself, or the gland perhaps has an ability to concentrate the diastase content. I doubt if the teleological explanation of the raison d'être of the diastase in human milk is right. Diastase, I suppose, plays a certain part in the complicated process of milk production in the cells of mammary glands and is secreted or excreted into milk as natural sequence, So. Grimmer ${ }^{10)}$ and Yoshiy a ${ }^{11)}$ supposed a participation of the diastase in the lactose production. I for myself want to see in the milk diastase a by-product in the milk production, because it is, as my result shows, rich in the milk nagative to A rakaw a's reaction i.e. in a " bad " milk.

Then why is the milk negative to Arakawa's reaction rich in diastase content? $\mathrm{Fuji}^{\mathrm{i}}{ }^{6)}$ in 1915 reported that the milk of infantile beriberi is rich in diastase content than the mean value of normal milk though it is poorer than colostrum is. The ratio, as he states, is :-Colostrum : beriberi milk : normal milk $=5: 3: 1$. This result seems to agree with my own, though at the time of his experiment the etiology of beriberi remained yet obscure. Moreover, he did not take the $\mathrm{pH}$ concentration into consideration in his experiment. (He diluted the milk with physiological $\mathrm{NaCl}$ solution) But generally speaking, the $\mathrm{pH}$ concentration of human milk is almost constant, and has a certain buffer action though it is smaller than that of cow's milk. We do not think his result was not largely influenced by $\mathrm{pH}$ concentration; his result will remain reliable for all that. $\mathrm{Chiba}^{7}$ investigated the variation of several constituents of milk by feeding 3 cows on B-avitaminotic fodder, but he could not reach any difinite conclusion as to the diastase content. This is very probably due to the very small amount of diastase in cow's milk as compared with human milk. Besides, he applied Wollg emu th's' ${ }^{12)}$ method of large ratio of dilution. Of

11) K.»Yoshiya, T. Onoy ama and A. Nakano, Zika Zassi, 1936, No. 432, 748.

12) J. Wohlgemuth, Biochem. Ztschr., 1908, 9, 1 and 10. 
late, $\mathrm{Sugi \textrm {e } ^ { 8 } )}$ reported an increase of milk diastase after vitamin $\mathrm{C}$ administration to the lactating mother. Is on ${ }^{13)}{ }^{14)}$, of our Pediatric Department, says in his papers that the milk negative to Arakawa's reaction contains much vitamin $C$ (or like substance), but that an administration of large amount of vitamin $C$ to the lactating mother will not influence the intensity of Ara$\mathrm{kaw}$ a's reaction remarkably. In view of these, my experiment seems to have much suggestion. Sa to ${ }^{15}$, of our Laboratcry, expressed that it is a general rule that diastase content in lactating women's urine is large in Arakawapositive cases and small in Arakawa-negative cases. It is already known that there is a decrease of blood and urine diastase and pancreas hypofunction in the case of berrberi or B-avitaminosis $\left(\mathrm{Mori}^{16)}, \mathrm{K} . \mathrm{Okada}^{17)}, \mathrm{T} . \mathrm{Okada}^{18)}\right.$, and Arimoto ${ }^{19)}$ etc.). Ottenstein ${ }^{20)}$ is of opinion that the great majority of blood diastase is originated from pancreas diastase. These statements are very interesting.

Now, it is well known that there are several moments which accelerate or retard enzymic action. My experiment was performed under constant $\mathrm{pH}$, constant temperature, and constant $\mathrm{NaC} 1$ concentration. But we notice the following reports of several authors. Of late, $\mathrm{Nakay} \mathrm{a} \mathrm{ma}{ }^{21)}$, in studying the influence of oxidation and reduction on enzymic action, saw that oxidative agent retards amylolytic action of the diastase and that reducing agent on the contrary accelerates it. He added a certain quantity of saturated $\mathrm{H}_{2} \mathrm{~S}$ solution into malt diastase solution so as to make its concentration be $1 / 1500-1 / 3000$ $\mathrm{mol}$ in the terminal solution and then amylolytic action of the enzyme was determined. He saw a remarkable accelerating action of $\mathrm{H}_{2} \mathrm{~S}$ on the diastase. This was especially remarkable in $\alpha$-amylase ( $\alpha$-diastase)*. Prior to his ex-. periment $\mathrm{Chrzascz}$ and Janicki22) reported in 1936 that, in adding $\mathrm{H}_{2} \mathrm{~S}$ to amylase which was extracted from barley, amylolytic action was largely accelerated. Pringshiem ${ }^{23}$ ) reported that glutathion and cystein accelerate the

13) S. Is o no, Tohoku J. Exp. Med., 1939, 35, 335.

14) S. Is o no, Tohoku J. Exp. Med., 1939, 35, 480.

15) Sh. Sato, Tohoku J. Exp. Med., 1940, 37, 392.

16) K. Mori, Nippon Shokakibyogakkai Zassi, 1912, 11, 211.

17) K. Okada, Kyoto-furitsu Ikadaigaku Zassi (Mitt. med. Akad. Kyoto), 1932, 6, 2705.

18) T. Okada, Aichi Igakkai Zassi, 1932, 29, 283.

19) K. A rimoto, Eiseigaku Densenbyogaku Zassi, 1932, 28, 302.

20) B. Ottenstein, Kl. Wschr., 1931, 10, 1261.

21) H. Na k a y ama, Igaku Kenkyu, 1939, 13, 297.

* In $1925 \mathrm{R}$. Kuhn (J. Li ebigs Annal. d. Chem., 1925, 443, 1.) reported that starch is splitted by some kind of amylase into two kinds of maltose, i.e. $\alpha$-maltose and $\beta$-maltose. Ku hn named the former as $a$-amylase, the latter $\beta$-amylase.

22) T. Chrzaszcz and J. Janick i, Biochem. Ztschr., 1936, 285, 47.

23) H. Pringsheim, H. Borchardt and H. Hupfer, Biochem. Ztschr., 1931, 238, 476. 
action of the pancreas and malt amylase. As is well known, glutathion and cystein have a certain reducing function. On the other hand, Taka mats $\mathrm{u}^{24)}$ of our Laboratory found methyl glyoxal (like substance) in the ether extract of the Arakawa-negative milk, but he was unable to identify it in the Arakawapositive milk at least in a large amount. Kasahara et al ${ }^{25)}$, Taniguchich and others reported that the Arakawa-negative milk contains much more vitamin $C$ than Arakawa-positive milk. Is on ${ }^{27)}{ }^{28)}$, of our Laboratory, says that the Arakawa-negative milk contains much amount of vitamin $C$ (or vitamin ( like substance) and that human milk has the antioxidative function, which is stronger in the Arakawa-negative milk than in Arakawa-positive milk. Methyl glyoxal and ascorbic acid are also regarded as reducing agents, and as above cited, Arakawa-negative milk has a strong antioxidative function. Hence it will be quite natural to say that the Arakawa-negative milk has a stronger reducing power than Arakawa-positive milk. But Stepp et al say, in their publication on the vitamins, that ascorbic acid retards vegetable $\beta$ amylase and pancreas amylase. On the contrary, as before cited, Sugie $\mathrm{e}^{8}$ saw an increase of milk diastase after vitamin Cadministration to lactating mothers. Though contrary to the opinion of Stepp et al ${ }^{29}$, it may be partly due to an increase of reducing power that the diastase is found rich in the milk after vitamin $\mathrm{C}$ administration. At any rate it is interesting that diastase is rich in the Arakawa-negative milk.

Further, diastase is much influenced by $\mathrm{NaC1}$, which accelerates the action of diastase. I shall here cite the results obtained by Nozaki and Ishii, who reported an increase of chlorine in Arakawa-negative milk. Of course, a difinite quantity of $\mathrm{NaCl}$ was added to the buffer solution in my experiment; and the amount of $\mathrm{NaC1}$ added was $450 \mathrm{mgrms} . \%$, so that the difference of $\mathrm{NaC1}$ between Arakawa-positive and -negative milk was negligible. Sammar-

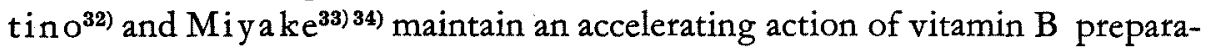
tion on diastase. But as my result shows, I failed to see such an action of vitamin $B$ (if pure crystalline $B_{1}$ used) on the milk diastase so far as my experi-

24) A. Takamatsu, Tohoku J. Exp. Med., 1934, 23, 46.

25) M. Kasahara, R. Kawamura, S. Takasu, J. Nagatoya, H. Fujii and S. Machi, Zika Zassi, 1935, No. 426, 1715.

26) T. Taniguchi, Zika Zassi, 1935, No. 426, 1665.

27) S. Is ono, Tohoku J. Exp. Med., 1939, 35, 335.

28) S. Is ono, Tohoku J. Exp. Med., 1939, 35, 480.

29) W. Stepp, J. Kühnau and H. Schroeder, Die Vitamine und ihre klinische Anwendung, 4th edit., 1939 Stüttgart, 179.

32) U. Sammartino, Biochem. Ztschr., 1921, 125, 25.

33) M. Miyake, Rinsho, 1924, 2, 290, 564 and 674.

34) M. Miyake, Nyujigaku Zassi, 1926, 1, 268. 
ment goes. I think that Miyake's result may be due partly to a relatively remarkable buffer action of the coarse vitamin B complex preparation and partly to its function as protective colloid. These may have acted to prevent the destruction of the enzyme and influence the change of $\mathrm{pH}$ concentration. Besides, coarse vitamin $\mathrm{B}$ preparation combines much iodine, hindering thus the colouration of iodine starch reaction. Miyake's result might perhaps be due to these factors. Contrary to his opinion, $I$ believe that vitamin $B_{1}$ is not a co-enzyme of diastase. If it were so as he says, the diastase might perhaps decrease more or less in the Arakawa-negative milk, while the fact is quite opposed to it.

Now I will discuss the result of my experiment. Taking into consideration what was described under Items 1-5 (Cf. Results of Experiment), it is clear that the milk negative to Arakawa's reaction is rich in diastase content though this shows a certain individual fluctuation. Generally and roughly, diastase content is reciprocally proportional to the intensity of Arakawa's reaction. If a milk sample is weaker in the intensity of the reaction, it is richer in diastase content, and if it is stronger in the reaction, then it is poorer in diastase content. But among a large numbers of milk samples, we can find a positive milk with a relatively high content of diastase and a negative milk with a low content, but such are rather exceptions.

With respect to the distribution of all the milk samples (Cf. Table III and IV) according to the diastase content and the intensity of Ara kawa's reaction, we see that the range of the distribution of diastase contents was relatively limited in the group of strong Arakawa's reaction, while in the group of the completely negative reaction the. range was very wide and was intermediate in the group of the weakly positive reaction. In other words, the curves of the Arakawa-positive group presents a very high, precipitious mountain and that of the weakly Arakawa-positive group a fairly high mountain, while that of the Arakawa-negative group a mountain chain or rather a hill-chain. The position of the peak of the mountain, which shows the most frequent diastase content in each group, is seen to shift to the right side with the weakening of Arakawa's reaction. (except one of the low peaks in the Arakawa-negative group.) The peak (a in Fig. 1) of the Arakawa-positive group is situated to the farthest right side. The fairly high peak (b in Fig. 1) of the weakly Arakawa-positive group is situated to the next farthest side, while the low peaks (c, d, e, f and $g$ in Fig. 1) of the completely Arakawa-negative grcup are for the most of them situated more or less farther to the left side. This shows that the diastase content is very constant in the cases of the strongly or normally Arakawa-positive group, very inconstant in the cases of the completely Arakawa-negative group, and of an intermediate constancy in the group of the 
weakly Arakawa-positive group.

As to the cases of one and the same lactants with different reaction on different sides of the breast, one can suppose that diastàse is probably rich on the weaker side of the breast. Very different Arakawa's reaction on different .sides of the breast in one and the same body means the possibility of better milk on one side and worse milk on the other side. This may seem absurd. The mechanism of milk production in the mammary gland is a very complicated one, though the details of it is not yet well known Prof. Sat ${ }^{35)}$ says in his paper that human milk is not a pure secretion, that it is physiologically a secretion plus filtration, and that it can be very frequently an excretion. In the majority of the cases of different A rak a wa's reaction in one and the same body, the fact that the diastase is rich in the milk of the side of the weaker reaction shows that diastase is closely connected to Arakaw a's reaction. Besides, in fact one lactant can secrete " good " and "bad" milk at the same time. Such is, for example, Case T.H. (No..133 and 134, Cf. Table VII). The mother told me that the infant used to vomit whenever she fed her infant with her right breast. Actually the milk from her right breast showed very weak A ra k a w o's reaction and a high value of diastase content. There is also an interesting report concernirg this problem outside of this country. Zappert and Jolles ${ }^{36)}$ reported and interesting case in twins, each accustomed to being fed exclusively from the definite side of the breast. The condition of one twin (girl who used to be fed from the left breast), who was weak and undernourished, improved when she was transferred to the right breast, while the other twin (boy who used to be fed from the right breast), who was hearty and strong, suffered nutritional impairment through such an exchange. This observation was accompanied by an analysis of the milk from both breasts which demonstrated that the milk of the left breast contained abundant fat and salt in it, which result is very interesting, if one learns the relation between fat and salts on one side and A raka wa's reaction on the other. I shall try to explain these facts in the light of A rak a wa's reaction. As before mentioned, human milk which is richer in fat content seems to show stronger diastatic activity. And as a result of studying fat content of human milk and its relation to A rakawa's reaction I have come to the conclusion* that Arakawa-nagative milk contains generally larger amount of fat than Arakawa-positive milk. Hence, it is with good reason to suppose that one case of a subject experienced by Zappert and Jol${ }^{l e s^{36)}}$ may very probably have been a case of a mother with strongly or nor-

35) A.Sato, Ky. Shoda and S. Miyazaki, Tohoku J. Exp. Med,, 1938, 34, 301.

36) J. Zappert and A. Jolles, Wien. med. Wschr., 1903, 53, 1914.

* That paper (176th Report of the Peroxidase Reaction) will be published in a coming issue of this Journal. . 
mally Arakawa-positive reaction (a "good" milk) on the right side, and completely Arakawa-negative reaction on the left side (a "bad" milk). If that case had been investigated with regards to diastase content, it might probably have been rich on the left side milk and poor in the right side milk.

In the cases one and the same lactant with equal Arakawa's reaction on different sides of the breast, $64 \%$ of the cases have a different diastase content, but the difference is always small except for a few cases, so that the general rule is that milk from both breasts is always of almost equal diastase content as far as Arakawa's reaction is equal in the intensity.

- The next subject is as to the diastase content of the milk secreted from " healthy " mothers with " healthy" sucklings. In the "Baby show" usually more or less well nourished babies appear. A rakawa's reaction of milk samples obtained in the "Baby show" will averagingly show a much better Ara$k$ awa's reaction than seen in our daily practice. So the mean value of diastase of the milk samples obtained in the "Baby show" is lower as compared with that obtained in our daily practice.

That diastase is contained very abundantly in colostrum is recognized by many workers (for example, Fujii' ${ }^{6}$, Chrzaszcz and Goralowana ${ }^{37}$, and Oshida $\left.{ }^{38}\right)$. Interesting is that even in colostrum with such a high value of diastase, a certain reverse proportion is seen between the content of the enzyme and the intensity of Arakawa's reaction. Kanayama ${ }^{39}$ reported the importance of A rakawa's reaction of colostrum as well as of milk in early period of lactation.

My result of the relation between the diastase content and the age of infants agrees with those of $\mathrm{Fujii}^{6}$ ) and of others. As has already been shown in the present paper, the content of milk diastase is different according to the intensity of A rakawa's reaction. It may not be right to compare, as to diastase content, milk samples of different lactation period among themselves. But I compared milk samples of equal intentisy of Arakawa's reaction among themselves under this precaution. I found that, if we observe the milk of the same age of infants, milk diastase is on an average always richer in the milk of weaker reaction without exception.

My result that younger mothers secrete more diastase into milk than older ones seems to agree with Fujii's ${ }^{6}$ report. He says that milk diastase was richer in the case of lactants who had given birth to less children. Char$z_{\left.a s z c z^{37}\right)}$ reported that a young cow's milk is richer in diastase content than older ones. Is it that the development of mammary glands in younger mothers

37) T. Chrzaszcz and C. Goralow an a, Biochem. Ztschr., 1935, 166, 172.

38) K. Oshida, Zika Zassi, 1933, No. 394, 575.

39) T. Kanay ama, Zika Zassi, 1931, No. 379, 1855. 
may possibly be still more or less inmature?

It is quite natural that milk obtained after feeding is richer in diastase content because our daily experience shows that Arakawa's reaction is apt to become more or less weaker after feeding. This is what has already been re-

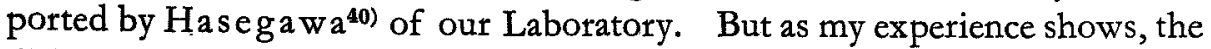
difference of diastase content before and after feeding is not so large unless Arakawa's reaction shows a remarkable change. To detect such a small difference, my method ${ }^{97}$ was especially convenient. Fujii ${ }^{6)}$ saw also richer diastase in milk after feeding. A similar phenomenon is observed in the salivary gland by Suzuki $\mathrm{i}^{\mathbf{1 1})}$ who saw that salivary diastase was richer after meal was taken, i.e. after a certain activity of the gland.

The milk from breasts with an anamnesis of mastitis or with actually affected with mastitis is rich in diastase content. Of course, mastitis milk is generally apt to react weakly to Arakawa's reaction, and so it is quite natural to expect a higher diastase content in such a milk, but sometimes mastitis milk shows rather a strong Arakawa's reaction. No. 1 and No. 3 show a strong reaction in spite of mastitis milk (Cf. Table XIV), but in case No. 1 milk of mastitis side has a remarkably high content of diastase, while in case No. 3 diastase of milk of the mastitis side is also richer than that of the healthy side milk, though the difference is small. Chrzaszcz ${ }^{37}$ ) and others reported that milk from cows affected with udder disease contained a larger amount of diastase, so that some authors are of opinion that the determination of milk diastase is of use for the early diagnosis of cow's mastitis.

In my experiment, the result shows that milk samples richer in fat content* seem to have richer diastase content, but as is well known milk fat content fluctuates remarkably due to a number of factors, so that it will be somewhat improper to say a decisive conclusion from the result of my present experiment alone. But Chrzaszcz and Goralowana ${ }^{37)}$ say that cow's milk richer in fat content is richer in diastase content.

In the cases of menstruation, pregnancy and syphilis, human milk seems to be rich in diastase content, and, as Arakawa's reaction is apt to be weakened in these cases, it is very probable. But as the number of my cases are still very few, I can not say anything conclusive. As to milk in menstruation I shall here cite the report by J. Steinert and G. Papp ${ }^{42)}$. They studied diastase

40) M. Hasegawa, Tohoku J. Exp. Med., 1937, 31, 491. 409.

41) T. Suzuki, Manshu Shikaigakkai Zassi, 1937, 11, 57. cit. Igakuchuo Zasshi, 1939, 62,

* As to the fat content of milk and its relation to A ra ka wa's reaction, I will report in this Journal in near future. (Cf. 176th Report of the Peroxidase Reaction).

42) J. Steinert and G. Pa p p, Ztschr. f. Kinderh., 1934, 56, 208. 
content of about 80 samplest $^{\dagger}$ of milk from 30 lactants and state that diastase content of milk sinks during the menstrual period and this is remarkable in the case of women who complain of severe pains. The result is quite contrary to my experience. But it is not to be excluded that his determination of diastaoo fell in the middle of menstrual period instaed of the premenstrual period, because the menstrual change seems to occur often in the premenstrual period as it was reported by Kokubo ${ }^{43}$ of our Laboratory (Cf. 27th Hematological Paper).

\section{Summary.}

Using my own diastase method ${ }^{9)}$, I estimated diastase content of human milk and investigated its relation to Arak awa's reaction. As a rule, human milk with weaker Arakawa's reaction is richer in diastase. As to the significance of the diastase in human milk, I presume that milk diastase may be a product or by-product necessary for milk production in the mammary gland and it is due to the disorder of the mammary gland caused by B-avitaminosis that Arakawa-negative milk contains a larger amount of diastase.

If I summarize the results of my experiment :-

1. Human milk shows a certain individual fluctuation in the diastase content. The mean value was 21.6.

2. The mean value of the diastase in the case of strong Arakawa's reaction was 14.8 . The mean value of diastase content in that of weakly positive reaction was 22.4 . The mean value of the diastase in that of completely negative reaction was 37.9 .

3. The diastase value over 15.0 was seen in $32 \%$ (about one third) of milk samples with strong A rakawa's reaction, in 69\% (about two thirds) of those of weakly positive reaction and in $84 \%$ (the large majority) of those of the completely Arakawa-negatire milk.

4. Milk diastase was richer on the weaker side of A rakawa's reaction in the case of one and the same lactant with different Arakawa's reaction on different sides of the breast.

5. There was no large fluctuation of diastase content in the case of one and the same lactant with equal Arakawa's reaction on both breasts.

6. The mean diastase value which is obtained in the "Baby show " was 14.1 for Arakawa-positive milk. 17.6 for Arakawa-negative milk and the average was 15.0 .

7. Colostrum was abundant in diastase.

I But no detailed data were given in their paper except in only one representative case.

43) Y. Kokubo, Tohoku J. Exp. Med., 1939, 35, 243. 
8. Milk diastase changes its value according to the age of sucklings. The diastase content, which is abundant in colostrum and in the milk of early lactations period, decreases gradually with the growing age of sucklings, but again it begins to increase when the time of weaning comes near.

9. The milk samples from younger mothers seemed to have richer diastase than older mothers.

10. Milk after feeding, contains richer diastase than before.

11. Milk from the breast affected with mastitis contains generally richer diastase.

12. Milk which is abundant in fat content seemed to have richer diastase.

13. Milk in the cases of menses, pregnancy and of syphilis seems to have richer diastase.

\section{Conclusion.}

In general human milk of weaker A rakawa's reaction contains richer. diastase than that of stronger A raka wa's reaction. Milk of strong or normal A rakawa's reaction contains small amount of diastase, and completely Arakawa-negative milk contains large amount of it. Milk of intermediate reactions contain diastase of intermediate amounts.

This work was carried out partly by a Grand from the Fund for Promotion of Scientific Research of the Education Department, for which we express here our thanks.

Prof. Akira Sato. 